\title{
CORRENTES EQUIVALENTES DE BORDA PARA A ANÁLISE DA RESPOSTA DE ANTENAS REFLETORAS EXCITADAS POR PULSOS
}

\author{
Cássio G. do Rego e Flavio J. V. Hasselmann
}

Resumo - Métodos assintóticos para altas frequências aplicados à determinação de formulações para correntes equivalentes de borda [1]-[4] foram revisados de forma compreensiva, o que permitiu a identificação de suas componentes de franja e de Ótica Física, bem como a eliminação de suas singularidades, levando à obtenção de expressões para o campo difratado válidas para pontos de observação com localização arbitrária, as quais podem ser aplicadas na análise de antenas refletoras, seja para a correção dos campos obtidos pela aplicação da Ótica Física (PO), ou para a determinação do campo difratado observado em pontos localizados sobre cáusticas [5]. Neste trabalho, estas expressões para os campos irradiados pelas correntes equivalentes são estendidas para $\mathrm{o}$ domínio do tempo mediante a aplicação de técnicas de inversão de Fourier e de uma representação analítica de sinais. A nova formulação assim obtida tem sua aplicabilidade atestada através da sua implementação na solução do problema da determinação da resposta de antenas refletoras convencionais de simetria axial, excitadas por fontes de comportamento temporal pulsado.

Palavras-chave: Antennas refletoras, análise no domínio do tempo, métodos assintóticos, Ótica Física, correntes equivalentes de borda.

Abstract - High-frequency asymptotic formulations for
equivalent edge currents [1]-[4] have been reviewed, allow-
ing the identification of fringe and Physical Optics compo-
nents and the extraction of their inherent singularities as well.
The resulting equations for the diffracted field are regular all
over the observation space and can be applied in the analy-
sis of reflector antennas either to correct the Physical Optics
formulation or to obtain the diffraction field near focal points
[5]. In this paper, the refered equations for the radiated field
are extended to the time domain using a Fourier inversion and
an analytical signal representation. The new temporal for-
mulation thus obtained is validated and has its applicability
acessed via the implementation in the solution of the tran-
sient response of conventional, circularly symmetric reflector
antennas excited by pulsed sources.

Keywords: Reflector antennas, time-domain analysis, asymptotic methods, Physical Optics, equivalent edge currents.

Este trabalho foi parcialmente financiado pelas seguintes agências de fomento à pesquisa: FAPEMIG, FAPERJ, CAPES e CNPq. Cássio G. do Rego é Professor Adjunto da UFMG, Departamento de Engenharia Eletrônica (e-mail: cassio@cpdee.ufmg.br), Flavio J. V. Hasselmann é Professor Associado do CETUC/PUC-Rio (e-mail: flavio@cetuc.puc-rio.br).

\section{INTRODUÇÃO}

Sistemas de comunicação sem fio de alta capacidade têm aplicação nos mais diversos serviços de telecomunicações da atualidade. A necessidade de acesso à internet em taxas cada vez mais elevadas, a implantação de novos sistemas de comunicação móvel pessoal multimídia e a convergência de vários tipos serviços têm promovido a pesquisa de esquemas de modulação que possibilitem o tráfego de bits em grandes quantidades. Neste contexto, sistemas faixa ultralarga (UWB) e rádio pulsado, bem como suas antenas associadas, têm sido estudados para oferecer uma alternativa interessante para prover um alto desempenho de capacidade de transmissão de informação. Isto é conseguido por meio da codificação das mensagens a serem transmitidas na forma de pulsos muito curtos que ocupam uma grande largura espectral, os quais são enviados diretamente através do canal rádio sem fazer uso de uma portadora senoidal como nos sistemas convencionais [6].

A análise da resposta de antenas refletoras excitadas por fontes de comportamento temporal pulsado, típicas de sistemas de alta capacidade, permite estudar o seu comportamento em faixas de frequências bastante largas. À medida em que as larguras de faixa ocupadas pelos sinais radioelétricos vêm aumentando, o que significa que estes são compostos por pulsos que vão se tornando mais estreitos, se faz necessário o emprego de novas técnicas de estudo para o seu espalhamento. Isto tem motivado o estudo do espalhamento de campos eletromagnéticos gerados por excitações pulsadas diretamente no domínio do tempo [7]-[15].

Existem vários métodos analíticos desenvolvidos para a análise de problemas de espalhamento de campos eletromagnéticos no domínio do tempo. A Teoria Espectral de Transientes (STT) foi desenvolvida para a determinação da solução exata para problemas de espalhamento considerandose geometrias canônicas [16]-[19]. Nesta técnica, o campo espalhado é expresso diretamente no domínio do tempo como uma integral espectral de ondas planas, a qual é resolvida analiticamente no plano complexo, onde se pode mostrar como as contribuições espectrais se adicionam para construir o campo. A solução exata para o problema canônico do espalhamento por uma cunha condutora de faces retas, considerando-se diferentes tipos de iluminação, foi obtida por Felsen a partir da técnica de expansão modal [20]. A despeito de serem exatas, as formulações obtidas nas soluções dos problemas canônicos mencionados são de difícil extensão para a aplicação em geometrias e excitações arbitrárias, pois apresentam uma dependência espaço-temporal implícita, 
o que dificulta a obtenção da resposta para o campo espalhado a partir de técnicas de convolução temporal [19].

Técnicas numéricas como o método das Diferenças Finitas no Domínio do Tempo (FDTD) e métodos de equações integrais no tempo e espaço têm sido aplicados na determinação da resposta transiente de espalhadores eletromagnéticos. Estas técnicas apresentam dificuldades como instabilidade, erros de interpolação, erros acumulados ao longo do tempo e a necessidade do uso de grandes quantidades de memória RAM e tempo de CPU quando aplicadas à solução de problemas que envolvam corpos espalhadores de grandes dimensões elétricas [15]. Este fato torna atraente o uso de versões temporais de técnicas analíticas assintóticas como a Ótica Física (TDPO) e o rastreamento de raios, representado pela Teoria Uniforme da Difração (TD-UTD) e pela Teoria Uniforme Assintótica (TD-UAT) [7]-[13]. É possível obter uma aproximação acurada para a resposta transiente do campo eletromagnético espalhado por objetos de forma complexa excitados por pulsos, da mesma forma que se faz no domínio da frequência, uma vez que não se dispõe de soluções exatas em forma analítica fechada. A simplicidade das correntes da Ótica Física sobre uma superfície suave e a versatilidade dos raios no domínio da frequência são mantidas no domínio do tempo, uma vez que uma função temporal pode ser expressa em termos de suas componentes espectrais [5]. No entanto, a exemplo do que acontece com formulações assintóticas de correntes induzidas e rastreamento de raios no domínio da frequência, existem certas limitações de aplicabilidade para a TDPO, TD-UTD e TD-UAT em problemas de antenas refletoras de simetria axial. No caso da TDPO, ocorrem imperfeições para o campo irradiado observado em pontos da região de lobos secundários, uma vez que as correntes da Ótica Física induzidas sobre superfícies condutoras são aproximadas em planos infinitos que as tangenciam. Sendo assim, as correntes próximas à borda, que contribuem majoritariamente para os campos difratados não são precisas e, portanto, devem ser corrigidas para permitir uma análise de desempenho adequada. Por outro lado, as técnicas de rastreamento de raios falham na determinação do campo em pontos de observação situados na proximidade de regiões onde há focalização de energia (cáusticas), típicas de antenas refletoras com simetria axial.

As limitações dos métodos assintóticos mencionadas acima podem ser contornadas a partir do uso de correntes equivalentes de borda, tanto para corrigir as correntes induzidas da Ótica Física, quanto para a determinação do campo difratado nas proximidades de cáusticas.

No presente artigo, a formulação das correntes equivalentes de borda no domínio da frequência [1]-[4] revisada recentemente [5], é estendida para o domínio do tempo por meio de uma inversão de Fourier e do uso de uma representação analítica de sinais temporais. As correntes equivalentes de borda no domínio do tempo assim obtidas são implementadas para a determinação dos campos difratados por antenas refletoras convencionais de simetria axial, excitadas por fontes de comportamento temporal pulsado. Para verificar a aplicabilidade e a precisão da formulação das correntes equivalentes, são determinados os campos difratados para pontos de observação localizados tanto em regiões próximas aos eixos de simetria dos refletores, quanto na região dos lobos secundários, e os resultados obtidos são comparados com aqueles determinados a partir de técnicas de rastreamento de raios e Ótica Física no domínio do tempo (TDPO). Todos os resultados obtidos da formulação apresentada aqui são validados através de sua comparação com aqueles resultantes a partir de uma solução de referência que se constitui da aplicação do Método dos Momentos no domínio da frequência, transformado para o domínio do tempo por uma inversão discreta de Fourier (MoM+IFFT).

Nos últimos anos, trabalhos publicados por Johansen [21], e Altintas e Russer [22] apresentaram correntes equivalentes de borda no domínio do tempo. No entanto, a formulação desenvolvida nestes trabalhos aplica-se à análise do espalhamento do campo eletromagnético por placas poligonais e discos condutores, não sendo adequada para levar em conta os efeitos de retardo de tempo associados às curvaturas do corpo espalhador. A formulação desenvolvida no domínio da frequência por Michaeli leva em conta as curvaturas dos espalhadores em regiões próximas às suas bordas [4], sendo, consequentemente, mais adequada para a aplicação em problemas de análise de antenas refletoras.

Este artigo está organizado como descrito a seguir. $\mathrm{Na}$ Seção 2 são apresentadas as técnicas para a inversão para o domínio do tempo da formulação de campos espalhados por superfícies condutoras no domínio espectral que serão utilizadas aqui [5]. É dada uma ênfase especial para a representação analítica de sinais, cuja importância e aplicabilidade serão evidenciadas. Também é discutida a relação entre os vetores do campo dos domínios do tempo e da frequência.

As expressões para os campos temporais irradiados por correntes equivalentes de borda são deduzidas na Seção 3 . A exemplo do que ocorre no domínio da frequência, as correntes equivalentes temporais também se escrevem como a soma de duas parcelas: correntes de franja e correntes da Ótica Física. A formulação para as correntes de franja é obtida a partir do uso de uma transformada inversa de Fourier, descrita na Seção 3.1, enquanto que a formulação para a parcela da Ótica Física é determinada pelo uso da representação analítica de sinais, descrita na Seção 3.2.

Na Seção 4 são tratadas as aplicações da formulação das correntes equivalentes de borda no domínio do tempo. A utilidade das expressões deduzidas neste trabalho é verificada, seja para corrigir os campos da TDPO em pontos de observação afastados da região do lobo principal, quanto para determinar o campo difratado em pontos de observação localizados próximos aos eixos de simetria de refletores focalizadores convencionais.

\section{FORMULAÇÕES PARA O DOMÍNIO DO TEMPO}

Esta seção mostra as técnicas utilizadas para a obtenção da formulação para o espalhamento de campos eletromagnéticos por superfícies condutoras no domínio do tempo, partindo-se de soluções conhecidas no domínio da frequência. 


\subsection{TRANSFORMADA INVERSA DE FOURIER}

Quando se conhece a formulação de uma técnica de solução de problemas de espalhamento no domínio da frequência, é possível usá-la para abordar problemas no domínio do tempo. Para tal, usa-se a transformada inversa de Fourier. Este procedimento tem a vantagem de poder ser utilizado diante de um novo problema no domínio do tempo que já tenha sido resolvido no domínio da frequência, podendo também ser utilizado como uma primeira abordagem.

A transformada inversa de Fourier, que será aplicada à determinação do campo espalhado em problemas no domínio do tempo, é dada por

$$
\vec{e}(\vec{r}, t)=\frac{1}{2 \pi} \int_{-\infty}^{\infty} \vec{E}(\vec{r}, \omega) e^{j \omega t} d \omega
$$

onde $\vec{E}(\vec{r}, \omega)$ representa a solução conhecida no domínio da frequência e $\omega$ é a frequência angular. A transformação acima pode ser escrita utilizando-se um operador $\mathcal{F}_{t}^{-1}$ :

$$
\vec{e}(\vec{r}, t)=\mathcal{F}_{t}^{-1}\{\vec{E}(\vec{r}, \omega)\},
$$

Escrevendo os campos elétricos no domínio da frequência na forma

$$
\vec{E}(\vec{r}, \omega)=\vec{E}_{0}(\vec{r}) F(\omega),
$$

onde $\vec{E}_{0}(\vec{r})$ determina o comportamento espacial dos campos e o comportamento espectral é coletado em $F(\omega)$, fica evidente, de (1), que

$$
\vec{e}(\vec{r}, t)=\vec{E}_{0}(\vec{r}) f(t),
$$

onde $f(t)$ e $F(\omega)$ formam um par de transformadas de Fourier:

$$
\begin{gathered}
f(t)=\frac{1}{2 \pi} \int_{-\infty}^{\infty} F(\omega) e^{j \omega t} d \omega=\mathcal{F}_{t}^{-1}\{F(\omega)\}, \\
F(\omega)=\int_{-\infty}^{\infty} f(t) e^{-j \omega t} d t=\mathcal{F}_{t}\{f(t)\} .
\end{gathered}
$$

Nem sempre é possível usar a transformada inversa de Fourier para se obter os campos no domínio do tempo diretamente a partir de seus correspondentes no domínio da frequência. Para a classe de funções $f(t)$ que satifaz às condições $f(t)=0$ para $t<0$ e $|f(t)|=O\left(e^{\alpha t}\right)$ para $t \rightarrow$ $\infty$, a convergência de (6) é assegurada forçando $\operatorname{Im}(\omega)>\alpha$. Neste caso, a equação (5) é expressa na forma

$$
f(t)=\frac{1}{2 \pi} \int_{-\infty+j \Delta}^{\infty+j \Delta} F(\omega) e^{j \omega t} d \omega
$$

onde $\Delta>\alpha$. O espectro $F(\omega)$ é calculado a partir de

$$
F(\omega)=\int_{0}^{\infty} f(t) e^{-j \omega t} d t,
$$

compreendendo uma função analítica para $\operatorname{Im}(\omega)>\alpha$. A equação (7) corresponde à transformada de Laplace, executada para a variável $s=j \omega$.

\section{REPRESENTAÇÃO ANALÍTICA DE FUNÇÕES TEMPORAIS}

Nesta sub-seção será apresentada a representação analítica de sinais e evidenciada sua relação com as transformadas de Fourier e Laplace.

\subsubsection{DEFINIÇÃO}

Seja $f(t)$ uma função temporal da variável real $t$, com transformada de Fourier $F(\omega)$ expressa por (6). Definese a representação analítica de $f(t)$ como a transformada de Fourier inversa de $2 U(\omega) F(\omega)$, onde $U(\omega)$ é o degrau unitário. Assim, define-se

$$
\begin{aligned}
+f(t)= & \frac{1}{2 \pi} \int_{-\infty}^{\infty} 2 U(\omega) F(\omega) e^{j \omega t} d \omega= \\
& \frac{1}{\pi} \int_{0}^{\infty} F(\omega) e^{j \omega t} d \omega, \operatorname{Im}(t)>0,
\end{aligned}
$$

onde se faz a continuação analítica da variável $t$ para o plano complexo, e a condição $\operatorname{Im}(t)>0$ garante que a função expressa em (9) é analítica na metade superior do plano $t$ e, consequentemente, a convergência da integral. Escrevendo a equação (9) com o auxílio de um operador, tem-se

$$
\stackrel{+}{f}(t)=\mathcal{A}_{\omega}\{F(\omega)\}
$$

Uma observação muito útil pode ser feita ao se analisar a representação analítica de sinais definida pela equação (9). Percebe-se que a integração tem a forma de uma transformada de Laplace, o que permite o uso extensivo de tabelas de transformadas existentes para se avaliar a representação analítica de sinais.

\subsubsection{PROPRIEDADES DA REPRESENTAÇÃO ANALÍTICA DE SINAIS}

Quando a função analítica $\stackrel{+}{f}(t)$ é avaliada no eixo real de $t, \operatorname{Im}(t)=0$, podemos escrever

$$
\begin{aligned}
+\stackrel{f}{f}(t) & =\frac{1}{2 \pi} \int_{-\infty}^{\infty} 2 U(\omega) F(\omega) e^{j \omega t} d \omega= \\
& =2 f(t) *\left[\frac{1}{2} \delta(t)-\frac{1}{j \pi t}\right]= \\
& =f(t)+\frac{j}{\pi} \mathrm{VP} \int_{-\infty}^{\infty} \frac{f(\tau)}{t-\tau} d \tau= \\
& =f(t)+j \mathcal{H}[f(t)],
\end{aligned}
$$

onde VP significa que um valor principal de Cauchy é obtido na integração de forma a excluir a singularidade em $\tau=t$, e $\mathcal{H}[f(t)]$ é a transformada de Hilbert da função temporal $f(t)$. Nota-se então que a função no tempo real $f(t)$ pode ser recuperada da função analítica fazendo $t$ real na equação (9) e tomando a parte real de $\stackrel{+}{f}(t)$. Sendo assim, um sinal analítico é um fasor no domínio do tempo, do qual se extrai o sinal 
real. Se a integral em (9) não converge quando $\operatorname{Im}(t)=0$, $\stackrel{+}{f}(t)$ não é analítica. Neste caso $\stackrel{+}{f}(t)$ em $\operatorname{Im}(t)=0$ é a distribuição definida por $\stackrel{+}{f}(t)$ em $\operatorname{Im}(t)>0$ na sua fronteira de analiticidade [23].

A transformada de Hilbert de $f(t)$ em (11) pode ser definida na forma de uma transforma inversa de Fourier, significando a aplicação de um retardo de fase de $\pi / 2$ radianos em todas as componentes espectrais de $f(t)$ [24]

$$
\mathcal{H}[f(t)]=\frac{1}{2 \pi} \int_{-\infty}^{\infty}-j \operatorname{sgn}(\omega) F(\omega) e^{j \omega t} d \omega
$$

o que permite usar os seguintes pares de transformadas de Fourier

$$
\begin{gathered}
\stackrel{+}{f}(t) \stackrel{\mathcal{F}}{\longleftrightarrow} 2 F(\omega) U(\omega), \\
f(t) \stackrel{\mathcal{F}}{\longleftrightarrow} F(\omega), \\
\mathcal{H}[f(t)] \stackrel{\mathcal{F}}{\longleftrightarrow}-j \operatorname{sgn}(\omega) F(\omega),
\end{gathered}
$$

e similarmente para $\stackrel{+}{g}(t), g(t)$ e $\mathcal{H}[g(t)]$, para deduzir as seguintes relações, válidas para $\operatorname{Im}(t)=0$ :

$$
\begin{gathered}
\frac{\partial}{\partial t} \stackrel{+}{f}(t) \stackrel{\mathcal{F}}{\longrightarrow} 2 j \omega F(\omega) U(\omega), \\
\int_{-\infty}^{\infty} g(t) f(t) d t=\frac{1}{2} \operatorname{Re}\left[\int_{-\infty}^{\infty} \stackrel{+}{f}(t) \stackrel{+}{g}(t) d t\right], \\
\mathcal{H}[f(t) * g(t)]=\mathcal{H}[f(t)] * g(t)=f(t) * \mathcal{H}[g(t)], \\
\mathcal{H}[f(t)] * \mathcal{H}[g(t)]=-f(t) * g(t), \\
\frac{1}{2} \stackrel{+}{f}(t) * \stackrel{+}{g}(t)=\stackrel{+}{f}(t) * g(t)=f(t) * \stackrel{+}{g}(t), \\
\frac{1}{2} \operatorname{Re}[\stackrel{+}{f}(t) * \stackrel{+}{g}(t)]=f(t) * g(t),
\end{gathered}
$$

onde $\bar{g}(t)$ é o complexo conjugado de $g(t)$ e $*$ representa uma convolução temporal:

$$
f(t) * g(t)=\int_{-\infty}^{\infty} f(\tau) g(t-\tau) d \tau
$$

\subsubsection{APLICAÇÃO DA REPRESENTAÇÃO ANALÍTICA DE SINAIS}

Quando se utiliza a transformada inversa de Fourier (1) é necessário conhecer o comportamento das soluções no domínio da frequência para todos os valores de $\omega$, de forma que, se o comportamento espectral do campo $\vec{E}(\vec{r}, \omega)$ é coletado em uma função $F(\omega)$, a relação $F(-\omega)=F^{*}(\omega)$ deve ser satisfeita para assegurar que a resposta obtida no domínio do tempo seja uma função real. Nos métodos assintóticos de análise do espalhamento de campos eletromagnéticos no domínio da frequência a formulação é desenvolvida para $\omega \rightarrow \infty$, fazendo com que haja a ocorrência de funções cujo comportamento espectral para $\omega<0$ não seja determinado, como por exemplo as funções de transição usuais nos métodos óticos. Como não é necessário conhecer as componentes de frequência negativas de $F(\omega)$ quando se usa a representação analítica de sinais (9), esta pode ser usada para se obter a solução temporal de problemas de espalhamento a partir de suas correspondentes no domínio da frequência:

$$
\vec{e}(\vec{r}, t)=\operatorname{Re}[\vec{e}(\vec{r}, t)], \operatorname{Im}(t)=0,
$$

onde

$$
\stackrel{+}{\vec{e}}(\vec{r}, t)=\mathcal{A}_{\omega}\{\vec{E}(\vec{r}, \omega)\} .
$$

\subsection{RELAÇÃO ENTRE OS CAMPOS NOS DOMÍNIOS TEMPORAL E ESPECTRAL NO REGIME DE ALTAS FREQUÊNCIAS}

O estudo do campo próximo a uma frente de onda dá um conhecimento adicional da relação entre os domínios do tempo e da frequência. Se o campo próximo a uma frente de onda é expresso na forma de uma série assintótica em $\omega$, no domínio da frequência, consegue-se determinar uma série assintótica em $t$ que descreve este campo no domínio do tempo.

Seja a expansão assintótica generalizada para os campos eletromagnéticos expressa em uma série de potências inversas de $\omega[25]$

$U(\vec{r}, \omega) \sim \exp \left[-j \omega \frac{S(\vec{r})}{c_{0}}\right]\left[A(\vec{r})+(j \omega)^{-\alpha-1} \sum_{m=0}^{\infty} \frac{U_{m}(\vec{r})}{(j \omega)^{m}}\right]$,

onde $A(\vec{r})$ e $U_{m}(\vec{r})$ são amplitudes dependentes das coordenadas espaciais, $c_{0}$ é a velocidade de propagação, $S(\vec{r})$ é a fase e o parâmetro $\alpha>-1$ é escolhido de acordo com a parcela de campo representada (Ótica Geométrica ou difratado). Quando (25) é continuada analiticamente, fazendo-se $\operatorname{Im}(\omega)<0$, e se usa a transformada inversa de Laplace (7) para se determinar uma expressão assintótica para o campo no domínio do tempo, obtém-se o resultado [26]

$$
\begin{aligned}
u(\vec{r}, t) \sim & A(\vec{r}) \delta\left(t-\tau_{0}\right)+ \\
& \left(t-\tau_{0}\right)^{\alpha} \sum_{m=0}^{\infty} \frac{U_{m}(\vec{r})}{\Gamma(\alpha+m+1)}\left(t-\tau_{0}\right)^{m}, t>\tau_{0},
\end{aligned}
$$

onde

$$
\tau_{0}=\frac{S(\vec{r})}{c_{0}}
$$

$\mathrm{e}$

$$
\Gamma(x)=\int_{0}^{\infty} y^{x-1} e^{-y} d y, \operatorname{Re}(x)>0 .
$$

A série de potências de $t$ obtida em (26) é assintótica para $t \rightarrow \tau_{0}$, significando que sua convergência para o termo dominante [primeiro termo de (26)] é maior para instantes de tempo próximos à chegada da primeira frente de onda ao ponto de observação, $t=\tau_{0}$. Sendo assim, conclui-se que as componentes de alta frequência do campo eletromagnético correspondem à parcela de energia que chega primeiro ao 
ponto de observação. Assim, as técnicas assintóticas no domínio do tempo, obtidas a partir da inversão de técnicas válidas para $\omega \rightarrow \infty$, têm sua validade restrita para $t \rightarrow \tau_{0}$ (early-time response).

\section{CORRENTES EQUIVALENTES NO DOMÍNIO DO TEMPO}

Na recente revisão da formulação das correntes equivalentes de borda no domínio espectral [5] foi mostrado que estas podem ser escritas como a soma de duas parcelas: uma delas apresenta uma distribuição de fase com comportamento não-uniforme à medida em que a superfície refletora é iluminada pelos campos incidentes, e corresponde à corrente de franja introduzida no trabalho de Ufimtsev [27], enquanto que a outra parcela é obtida a partir das correntes da Ótica Física nas proximidades da borda do espalhador, sendo por este motivo denominada de componente da PO. Cada uma destas parcelas apresenta, a princípio, singularidades nas regiões das fronteiras de sombra e reflexão, as quais são extraídas, de maneira diferente para cada uma das parcelas, de forma a se obter uma formulação que seja válida para qualquer aspecto de observação. Lembrando que o campo irradiado pelas correntes de borda corresponde ao campo difratado pela borda do espalhador quando se usa técnicas de rastreamento de raios, e considerando cada uma das parcelas identificadas acima, observa-se que é possível utilizar as correntes de franja para se fazer uma correção do campo espalhado por condutores elétricos perfeitos, quando este é determinado a partir de correntes superficiais da Ótica Física.

O objetivo a ser alcançado nesta seção é fazer a extensão dos campos irradiados pelas correntes equivalentes para o domínio do tempo. Como o interesse de aplicação da formulação a ser obtida é direcionado para a análise da resposta temporal de antenas refletoras, será apresentada a versão temporal da Ótica Física (TDPO), técnica de correntes induzidas introduzida por Sun e Rusch [15]. A seguir, as correntes de franja no domínio do tempo, que têm aplicação na correção das correntes da TDPO, são deduzidas através da utilização da transformada inversa de Fourier da formulação correspondente no domínio da frequência. Para se obter a versão temporal das correntes da parcela da PO que, somadas às correntes de franja, possibilitam a determinação do campo difratado, é necessário utilizar a representação analítica de sinais em (9), pois a formulação com singularidades extraídas, correspondente a esta parcela no domínio da frequência, envolve uma função de transição que não é definida para $\omega<0$ [5, Eq. (4.145)].

\section{1 ÓTICA FÍSICA NO DOMÍNIO DO TEMPO}

A técnica da TDPO é desenvolvida através de uma inversão de Fourier [Eqs. (1) e (2)] da formulação correspondente no domínio da frequência. Os campos distantes espalhados por uma superfície refletora iluminada por um campo magnético

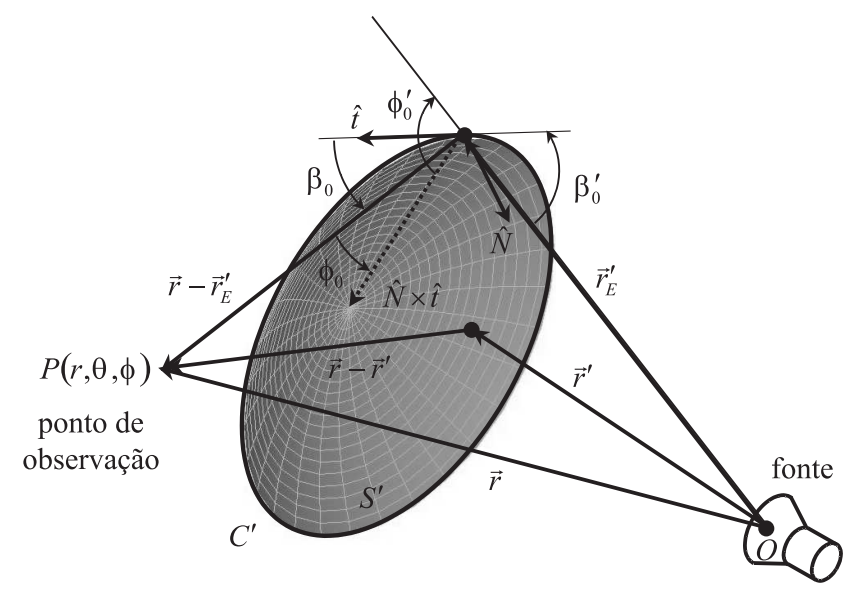

Figura 1. Parâmetros geométricos para a determinação das correntes induzidas sobre uma superfície refletora.

incidente $\vec{h}^{i}(\vec{r}, t)$ são representados pelos vetores [14, 15]

$$
\begin{gathered}
\vec{e}^{T D P O}(\vec{r}, t)=-\frac{\eta_{0}}{4 \pi r c} \int_{S^{\prime}} \frac{\partial \vec{j}_{\perp}^{P O}\left(\vec{r}^{\prime}, \tau\right)}{\partial \tau} d S^{\prime}, \\
\vec{h}^{T D P O}(\vec{r}, t)=\frac{\hat{r}}{\eta_{0}} \times \vec{e}^{T D P O}(\vec{r}, t) .
\end{gathered}
$$

Nas expressões acima tem-se a distribuição de correntes superficiais induzidas no refletor

$$
\vec{j}_{\perp}^{P O}\left(\vec{r}^{\prime}, \tau\right)=\vec{j}_{S}^{P O}\left(\vec{r}^{\prime}, \tau\right)-\left[\vec{j}_{S}^{P O}\left(\vec{r}^{\prime}, \tau\right) \cdot \hat{r}\right] \hat{r},
$$

com

$$
\vec{j}_{S}^{P O}\left(\vec{r}^{\prime}, \tau\right)=2 \hat{N} \times \vec{h}^{i}\left(\vec{r}^{\prime}, \tau\right),
$$

e o retardo de tempo associado à iluminação da superfície e a radiação subsequente das correntes induzidas

$$
\tau=t-\frac{\left|\vec{r}-\vec{r}^{\prime}\right|}{c} .
$$

Nas equações apresentadas $\eta_{0}$ é a impedância intrínseca do espaço livre, $c$ é a velocidade da luz no vácuo, o vetor $\vec{r}$ determina o ponto de observação dos campos e $\vec{r}^{\prime}$ localiza o ponto de integração sobre a superfície $S^{\prime}$ do refletor. Os vetores posição são ilustrados na Fig. 1.

\subsection{CORRENTES DE FRANJA NO DOMÍNIO DO TEMPO}

A Fig. 1 mostra os vetores associados à formulação das correntes equivalentes de borda em uma superfície refletora no domínio do tempo: $\vec{r}_{E}^{\prime}$ que localiza as correntes ao longo da borda $C^{\prime}, \vec{r}-\vec{r}_{E}^{\prime}$ que localiza a posição relativa entre entre um ponto da borda e o ponto de observação, e o vetor tangente unitário $\hat{t}$. Os vetores e parâmetros geométricos inerentes à sua determinação são calculados da mesma maneira, seja tanto no domínio do tempo quanto no domínio da frequência [5].

O campo irradiado pelas correntes de franja no domínio do tempo que será somado àquele da TDPO, expresso em (29), 
pode ser obtido a partir da inversão de Fourier da equação (4.162) da Ref. [5]:

$$
\vec{e}^{F W C}(\vec{r}, t)=\mathcal{F}_{t}^{-1}\left\{\vec{E}^{F W C}(\vec{r}, \omega)\right\} .
$$

Fazendo uso das expressões (4.109) e (4.110) da Ref. [5] para as correntes de franja no domínio da frequência, com as singularidades associadas à observabilidade no entorno das fronteiras de sombra e de reflexão eliminadas, na equação acima, e observando que os coeficientes angulares $D_{h}^{M, f}\left(\vec{r}^{\prime}, \vec{r}_{E}^{\prime}\right)$, $D_{e}^{I, f}\left(\vec{r}^{\prime}, \vec{r}_{E}^{\prime}\right)$ e $D_{h}^{I, f}\left(\vec{r}^{\prime}, \vec{r}_{E}^{\prime}\right)$ [5, Eqs. (4.111)-(4.113)] só assumem valores reais e não dependem da frequência angular $\omega$, pode-se escrever

$$
\begin{aligned}
& \vec{e}^{F W C}(\vec{r}, t)=\frac{-1}{4 \pi r} \int_{C^{\prime}}\left\{\left[\mathcal{F}_{\tau_{E}}^{-1}\left\{\vec{E}^{i}\left(\vec{r}_{E}^{\prime}, \omega\right) \cdot \hat{t}\right\} D_{e}^{I, f}\left(\vec{r}^{\prime}, \vec{r}_{E}^{\prime}\right)\right.\right. \\
& \left.+\mathcal{F}_{\tau_{E}}^{-1}\left\{\vec{H}^{i}\left(\vec{r}_{E}^{\prime}, \omega\right) \cdot \hat{t}\right\} \eta_{0} D_{h}^{I, f}\left(\vec{r}^{\prime}, \vec{r}_{E}^{\prime}\right)\right][\hat{t}-(\hat{t} \cdot \hat{r}) \hat{r}] \\
& \left.-(\hat{r} \times \hat{t}) \mathcal{F}_{\tau_{E}}^{-1}\left\{\vec{H}^{i}\left(\vec{r}_{E}^{\prime}, \omega\right) \cdot \hat{t}\right\} \eta_{0} D_{h}^{M, f}\left(\vec{r}^{\prime}, \vec{r}_{E}^{\prime}\right)\right\} d \ell^{\prime}
\end{aligned}
$$

onde $\vec{E}^{i}\left(\vec{r}_{E}^{\prime}, \omega\right)$ e $\vec{H}^{i}\left(\vec{r}_{E}^{\prime}, \omega\right)$ denotam o campo eletromagnético fasorial incidente sobre a borda e, de acordo com a notação utilizada na definição da transformada de Fourier em (1), tem-se

$$
\mathcal{F}_{\tau_{E}}^{-1}\{F(\omega)\}=\frac{1}{2 \pi} \int_{-\infty}^{\infty} F(\omega) e^{j \omega \tau_{E}} d \omega
$$

com o retardo de tempo devido à incidência sobre a borda do espalhador e posterior radiação das correntes equivalentes até o ponto de observação sendo expresso por

$$
\tau_{E}=t-\frac{\left|\vec{r}-\vec{r}_{E}^{\prime}\right|}{c}
$$

Sendo assim, obtém-se

$$
\begin{aligned}
\vec{e}^{F W C}(\vec{r}, t)= & \frac{-1}{4 \pi r} \int_{C^{\prime}}\left\{\eta_{0} \frac{\partial i^{f}\left(\vec{r}_{E}^{\prime}, t\right)}{\partial t}[\hat{t}-(\hat{t} \cdot \hat{r}) \hat{r}]-\right. \\
& \left.\frac{\partial m^{f}\left(\vec{r}_{E}^{\prime}, t\right)}{\partial t}(\hat{r} \times \hat{t})\right\} d \ell^{\prime}
\end{aligned}
$$

onde

$$
\begin{aligned}
\frac{\partial i^{f}\left(\vec{r}_{E}^{\prime}, t\right)}{\partial t}= & -\frac{1}{\eta_{0}}\left[\vec{e}^{i}\left(\vec{r}_{E}^{\prime}, \tau_{E}-\frac{\left|\vec{r}_{E}^{\prime}\right|}{c}\right) \cdot \hat{t}\right] D_{e}^{I, f}\left(\vec{r}^{\prime}, \vec{r}_{E}^{\prime}\right)+ \\
& {\left[\vec{h}^{i}\left(\vec{r}_{E}^{\prime}, \tau_{E}-\frac{\left|\vec{r}_{E}^{\prime}\right|}{c}\right) \cdot \hat{t}\right] D_{h}^{I, f}\left(\vec{r}^{\prime}, \vec{r}_{E}^{\prime}\right), } \\
\frac{\partial m^{f}\left(\vec{r}_{E}^{\prime}, t\right)}{\partial t}= & -\eta_{0}\left[\vec{h}^{i}\left(\vec{r}_{E}^{\prime}, \tau_{E}-\frac{\left|\vec{r}_{E}^{\prime}\right|}{c}\right) \cdot \hat{t}\right] D_{h}^{M, f}\left(\vec{r}^{\prime}, \vec{r}_{E}^{\prime}\right),
\end{aligned}
$$

representam as derivadas temporais das correntes de franja elétrica, $i^{f}\left(\vec{r}_{E}^{\prime}, t\right)$, e magnética, $m^{f}\left(\vec{r}_{E}^{\prime}, t\right)$, obtidas a partir da inversão de Fourier de seus correspondentes no domínio da frequência [5, Eqs. (4.109) e (4.110)]. Nas equações (39) e (40), $\vec{e}^{i}\left(\vec{r}_{E}^{\prime}, \tau_{E}-\frac{\left|\vec{r}_{E}^{\prime}\right|}{c}\right)$ e $\vec{h}^{i}\left(\vec{r}_{E}^{\prime}, \tau_{E}-\frac{\left|\vec{r}_{E}^{\prime}\right|}{c}\right)$ são os campos incidentes sobre a borda da superfície com o retardo de tempo adequado para se levar em conta a propagação do campo irradiado até o ponto de observação.

Ainda, para aplicar a equação (38) na correção do (em adição ao) campo da TDPO, expresso em (29), a problemas de espalhamento por superfícies condutoras com borda, fazse $n=2$ nas expressões dos coeficientes angulares das correntes de franja, expressos pelas Eqs. (4.111)-(4.113) da Ref. [5]. Este procedimento leva ao resultado

$$
D_{e}^{I, f}\left(\vec{r}^{\prime}, \vec{r}_{E}^{\prime}\right)=\frac{\operatorname{sen}\left(\phi_{0}^{\prime} / 2\right)}{\operatorname{sen}^{2} \beta_{0}^{\prime}} g\left(\phi_{0}^{\prime}, \alpha_{E}\right),
$$

$$
\begin{aligned}
D_{h}^{I, f}\left(\vec{r}^{\prime}, \vec{r}_{E}^{\prime}\right)= & \left\{\frac{\cot \beta_{0}^{\prime}\left[1+2 \operatorname{sen}\left(\alpha_{E} / 2\right) \cos \left(\phi_{0}^{\prime} / 2\right)\right]}{\operatorname{sen} \beta_{0}^{\prime} \operatorname{sen}\left(\alpha_{E} / 2\right)}-\right. \\
& \left.\frac{\cot \beta_{0} \cos \phi_{0}}{\operatorname{sen} \beta_{0}^{\prime} \operatorname{sen}\left(\alpha_{E} / 2\right)}\right\} g\left(\phi_{0}^{\prime}, \alpha_{E}\right), \\
D_{h}^{M, f}\left(\vec{r}^{\prime}, \vec{r}_{E}^{\prime}\right)= & \frac{\operatorname{sen} \phi_{0}}{\operatorname{sen} \beta_{0} \operatorname{sen} \beta_{0}^{\prime} \operatorname{sen}\left(\alpha_{E} / 2\right)} g\left(\phi_{0}^{\prime}, \alpha_{E}\right),
\end{aligned}
$$

onde

$$
\begin{gathered}
g\left(\phi_{0}^{\prime}, \alpha_{E}\right)=\frac{1}{\left[\cos \left(\phi_{0}^{\prime} / 2\right)+\operatorname{sen}\left(\alpha_{E} / 2\right)\right]}, \\
\alpha_{E}=\cos ^{-1}\left[\frac{\operatorname{sen} \beta_{0} \cos \phi_{0}+\cot \beta_{0}^{\prime}\left(\cos \beta_{0}-\cos \beta_{0}^{\prime}\right)}{\operatorname{sen} \beta_{0}^{\prime}}\right] .
\end{gathered}
$$

Os ângulos $\beta_{0}^{\prime}, \phi_{0}^{\prime}$, $\beta_{0}$ e $\phi_{0}$, ilustrados na Fig. 1, são obtidos a partir dos vetores $\vec{r}_{E}^{\prime}, \vec{r}, \hat{t}$ e do unitário normal à aresta, $\hat{N}$. Eles são determinados a partir das expressões

$$
\begin{aligned}
& \cos \beta_{0}^{\prime}=\frac{\vec{r}_{E}^{\prime}}{\left|\vec{r}_{E}^{\prime}\right|} \cdot \hat{t}, \\
& \cos \beta_{0}=\frac{\vec{r}}{|\vec{r}|} \cdot \hat{t},
\end{aligned}
$$

$$
\begin{aligned}
\cos \phi_{0}^{\prime}= & \frac{-\vec{r}_{E}^{\prime} \cdot(\hat{N} \times \hat{t})}{\left|\vec{r}_{E}^{\prime}\right| \operatorname{sen} \beta_{0}^{\prime}}, \\
\operatorname{sen} \phi_{0}^{\prime} & =\frac{-\vec{r}_{E}^{\prime} \cdot \hat{N}}{\left|\vec{r}_{E}^{\prime}\right| \operatorname{sen} \beta_{0}^{\prime}}, \\
\cos \phi_{0} & =\frac{\vec{r} \cdot(\hat{N} \times \hat{t})}{|\vec{r}| \operatorname{sen} \beta_{0}}, \\
\operatorname{sen} \phi_{0} & =\frac{\vec{r} \cdot \hat{N}}{|\vec{r}| \operatorname{sen} \beta_{0}} .
\end{aligned}
$$

\subsection{CORRENTES EQUIVALENTES DE BORDA NO DOMÍNIO DO TEMPO}

De posse da formulação das correntes de franja no domínio do tempo, com derivadas representadas em (39) e (40) e cujo campo radiado é expresso em (38), é possível se determinar o campo difratado em pontos de observação localizados sobre cáusticas. Para isso, é necessário adicionar-se às correntes de franja uma parcela uniforme associada ao campo da PO, o que resulta em correntes equivalentes de borda 
que irradiam o campo eletromagnético equivalente ao campo difratado obtido por rastreamento ótico.

Para se obter o campo irradiado por estas correntes equivalentes de borda no domínio do tempo para cunhas curvas e/ou superfícies com bordas, é necessário determinar a versão temporal da parcela uniforme obtida a partir das correntes da PO nas proximidades da borda. O comportamento espectral da função de Fresnel modificada, empregada para tornar regular a parcela uniforme das correntes equivalentes em pontos de observação sobre fronteiras de sombra e reflexão, $F_{e c}(\omega)$, expressa na equação (4.145) da Ref. [5], não é definido para $\omega<0$, obrigando o uso da representação analítica de sinais para se proceder à inversão da formulação do domínio da frequência para o domínio do tempo. Assim, aplicando a representação analítica de sinais definida pelas equações (9) e (10) ao campo irradiado expresso em [5, equação (4.169)], obtémse

$$
\begin{aligned}
& \stackrel{+}{\vec{e}^{P O C}}(\vec{r}, t)=\mathcal{A}_{\omega}\left\{\vec{E}^{P O C}(\vec{r}, \omega)\right\}= \\
& \frac{-1}{4 \pi r c} \int_{C^{\prime}}\left\{\left[\mathcal{A}_{\omega}\left\{j \omega F_{e c}(\omega)\left[\vec{E}^{i}\left(\vec{r}_{E}^{\prime}, \omega\right) \cdot \hat{t}\right]\right\} D_{e}^{I, P O}\left(\vec{r}^{\prime}, \vec{r}_{E}^{\prime}\right)\right.\right. \\
& \left.+\mathcal{A}_{\omega}\left\{j \omega F_{e c}(\omega)\left[\vec{H}^{i}\left(\vec{r}_{E}^{\prime}, \omega\right) \cdot \hat{t}\right]\right\} \eta_{0} D_{h}^{I, P O}\left(\vec{r}^{\prime}, \vec{r}_{E}^{\prime}\right)\right] \\
& {[\hat{t}-(\hat{t} \cdot \hat{r}) \hat{r}]+(\hat{r} \times \hat{t}) \mathcal{A}_{\omega}\left\{j \omega F_{e c}(\omega)\left[\vec{H}^{i}\left(\vec{r}_{E}^{\prime}, \omega\right) \cdot \hat{t}\right]\right\}} \\
& \left.\eta_{0} D_{h}^{M, P O}\left(\vec{r}^{\prime}, \vec{r}_{E}^{\prime}\right)\right\} d \ell^{\prime},
\end{aligned}
$$

com a integral de linha acima sendo efetuada ao longo da borda da superfície refletora.

Fazendo uso das propriedades de derivação temporal (16) e convolução (21) da representação analítica de sinais, chegase ao resultado

$$
\begin{aligned}
& \vec{e}^{\stackrel{+}{P O C}}(\vec{r}, t)=\frac{-1}{4 \pi r} \int_{C^{\prime}}\left\{\eta_{0} \frac{\partial i^{P O}\left(\vec{r}_{E}^{\prime}, t\right)}{\partial t}[\hat{t}-(\hat{t} \cdot \hat{r}) \hat{r}]-\right. \\
& \left.\frac{\partial m^{+}{ }^{+}\left(\vec{r}_{E}^{\prime}, t\right)}{\partial t}(\hat{r} \times \hat{t})\right\} d \ell^{\prime}
\end{aligned}
$$

onde $i^{P O}\left(\vec{r}_{E}^{\prime}, t\right)$ e $m^{P O}\left(\vec{r}_{E}^{\prime}, t\right)$ são as parcelas uniformes das correntes de borda, elétrica e magnética, cujas representações analíticas de suas derivadas temporais são obtidas a partir das Eqs. (4.154) e (4.155) da Ref. [5], resultando

$$
\begin{aligned}
& \frac{\partial i^{P O}\left(\vec{r}_{E}^{\prime}, t\right)}{\partial t}= \\
& -\frac{1}{\eta_{0}} \frac{\partial}{\partial t}\left\{\stackrel{+}{f}_{e c}(t) *\left[\vec{e}^{i}\left(\vec{r}_{E}^{\prime}, \tau_{E}-\frac{\left|\vec{r}_{E}^{\prime}\right|}{c}\right) \cdot \hat{t}\right]\right\} D_{e}^{I, P O}\left(\vec{r}^{\prime}, \vec{r}_{E}^{\prime}\right) \\
& +\frac{\partial}{\partial t}\left\{\stackrel{+}{f}_{e c}(t) *\left[\vec{h}^{i}\left(\vec{r}_{E}^{\prime}, \tau_{E}-\frac{\left|\vec{r}_{E}^{\prime}\right|}{c}\right) \cdot \hat{t}\right]\right\} D_{h}^{I, P O}\left(\vec{r}^{\prime}, \vec{r}_{E}^{\prime}\right),
\end{aligned}
$$

$$
\begin{aligned}
& \frac{\partial m^{P} O\left(\vec{r}_{E}^{\prime}, t\right)}{\partial t}= \\
& -\eta_{0} \frac{\partial}{\partial t}\left\{\stackrel{+}{f}_{e c}(t) *\left[\vec{h}^{i}\left(\vec{r}_{E}^{\prime}, \tau_{E}-\frac{\left|\vec{r}_{E}^{\prime}\right|}{c}\right) \cdot \hat{t}\right]\right\} D_{h}^{M, P O}\left(\vec{r}^{\prime}, \vec{r}_{E}^{\prime}\right),
\end{aligned}
$$

$\operatorname{com} D_{h}^{M, P O}\left(\vec{r}^{\prime}, \vec{r}_{E}^{\prime}\right), D_{e}^{I, P O}\left(\vec{r}^{\prime}, \vec{r}_{E}^{\prime}\right)$ e $D_{h}^{I, P O}\left(\vec{r}^{\prime}, \vec{r}_{E}^{\prime}\right)$ sendo expressos por

$$
\begin{gathered}
D_{h}^{M, P O}\left(\vec{r}^{\prime}, \vec{r}_{E}^{\prime}\right)=-\frac{2 \operatorname{sen} \phi_{0}}{\operatorname{sen} \beta_{0}} \\
D_{e}^{I, P O}\left(\vec{r}^{\prime}, \vec{r}_{E}^{\prime}\right)=\frac{2 \operatorname{sen} \phi_{0}^{\prime}}{\operatorname{sen} \beta_{0}^{\prime}} \\
D_{h}^{I, P O}\left(\vec{r}^{\prime}, \vec{r}_{E}^{\prime}\right)=-2\left(\cot \beta_{0}^{\prime} \cos \phi_{0}^{\prime}+\cot \beta_{0} \cos \phi_{0}\right) .
\end{gathered}
$$

Nas Eqs. (54) e (55) são as utilizadas as representações analíticas dos campos incidentes com o retardo de tempo adequado para se levar em conta a propagação até o ponto de observação, e o sinal $*$ denota uma integral de convolução temporal, como em (22).

A função de Fresnel modificada para o domínio do tempo, $\stackrel{+}{f}_{e c}(t)$, é determinada a partir da representação analítica de sinais, obtendo-se

$$
\begin{aligned}
\stackrel{+}{f}_{e c}(t)= & \frac{1}{\pi} \int_{0}^{\infty} F_{e c}(\omega) e^{j \omega t} d \omega= \\
& -\frac{2 j B_{1}}{\pi} \frac{\operatorname{arctanh}\left(\sqrt{1+t / B_{2}^{2}}\right)}{B_{2} \sqrt{1+t / B_{2}^{2}}}, \operatorname{Im}(t) \geq 0,
\end{aligned}
$$

onde $B_{1}$ e $B_{2}$ são dados pelas equações (4.149) e (4.150) da Ref. [5], respectivamente.

O campo irradiado pela parcela uniforme das correntes de borda é, então, extraído de sua representação analítica em (53), a partir de (23), na forma

$$
\vec{e}^{P O C}(\vec{r}, t)=\operatorname{Re}\left[\vec{e}^{+}{ }^{+}(\vec{r}, t)\right], \operatorname{Im}(t)=0 .
$$

\subsection{CAMPOS IRRADIADOS}

De posse das parcelas de campo irradiado pelas correntes equivalentes para superfícies com borda, expressas em (38) e (60), obtém-se o campo irradiado pelas correntes equivalentes de borda sem singularidades, para quaisquer aspectos de observação:

$$
\vec{e}^{E E C}(\vec{r}, t)=\vec{e}^{F W C}(\vec{r}, t)+\vec{e}^{P O C}(\vec{r}, t),
$$

o qual pode ser utilizado como alternativa ao cálculo do campo difratado em pontos de observação situados sobre cáusticas. 


\section{APLICAÇÕES: ANÁLISE DE ANTE- NAS REFLETORAS CONVENCIONAIS COM SIMETRIA AXIAL}

Nesta seção, a formulação do campo irradiado pelas correntes equivalentes de borda é utilizada para a determinação do campo espalhado por antenas refletoras convencionais. A validação desta técnica de análise é feita comparando-se os resultados numéricos obtidos com a solução de referência MoM+IFFT. Lembrando aqui que as técnicas de análise de espalhamento de campos eletromagnéticos desenvolvidas ao longo deste trabalho são assintóticas, é interessante se fazer uma comparação dos resultados obtidos a partir de sua aplicação com aqueles fornecidos através da aplicação de técnicas de rastreamento de raios, como a versão temporal da Teoria Uniforme da Difração (TD-UTD). A exemplo do que ocorre com a sua correspondente no domínio da frequência, o campo da TD-UTD pode ser escrito como a soma de duas parcelas: uma associada ao campo da Ótica Geométrica (TD-GO) e outra ao campo difratado modificado [5, 11, 23].

\subsection{HIPERBOLÓIDE ILUMINADO PELO FOCO}

O primeiro caso de estudo a ser examinado envolve a determinação dos campos espalhados por um hiperbolóide de revolução com diâmetro de 7,5 m e excentricidade $e=2,3$, iluminado a partir do foco por um pulso duplo-gaussiano, o qual é um sinal banda larga sem componente DC [5, 11], como pode ser observado na Fig. 2, onde sua forma de onda e suas componentes espectrais são mostradas, sendo possível se observar que as frequências mais significativas, isto é, as componentes espectrais de maior amplitude, estão distribuídas em torno de $500 \mathrm{MHz}$. O comportamento temporal da excitação tem a forma

$$
f(t)=4 \exp \left\{-\left[4\left(\frac{c}{a}\right) t\right]^{2}\right\}-2 \exp \left\{-\left[2\left(\frac{c}{a}\right) t\right]^{2}\right\}
$$

onde o coeficiente $a$ determina a largura do pulso. Para o caso em estudo foi utilizado o valor $a=0,6 \mathrm{~m}$, o que implica em um pulso de largura de aproximadamente $5 \mathrm{~ns}$.

O campo incidente no refletor é uma onda esférica, com lei de iluminação $\cos ^{n} \theta_{F}$, expresso por

$\vec{e}^{i}\left(\vec{r}_{F}, t\right)=\frac{f\left(t-\tau_{F}\right)}{\left|\vec{r}_{F}\right|} \cos ^{n} \theta_{F}\left(\cos \phi_{F} \hat{a}_{\theta_{F}}-\operatorname{sen} \phi_{F} \hat{a}_{\phi_{F}}\right)$,

com

$$
\tau_{F}=\frac{\left|\vec{r}_{F}\right|}{c}
$$

onde o índice $F$ referencia o sistema de coordenadas esféricas com origem no centro de fase do alimentador (ponto $O$ na Fig. 1) e equivale ao super-escrito / nas expressões de campo na seção anterior. O comportamento temporal regido por $f(t)$ é expresso em (62).

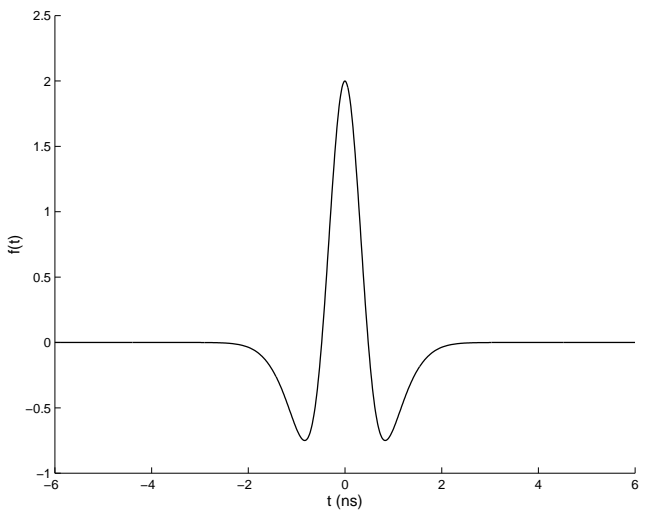

(a) Comportamento temporal

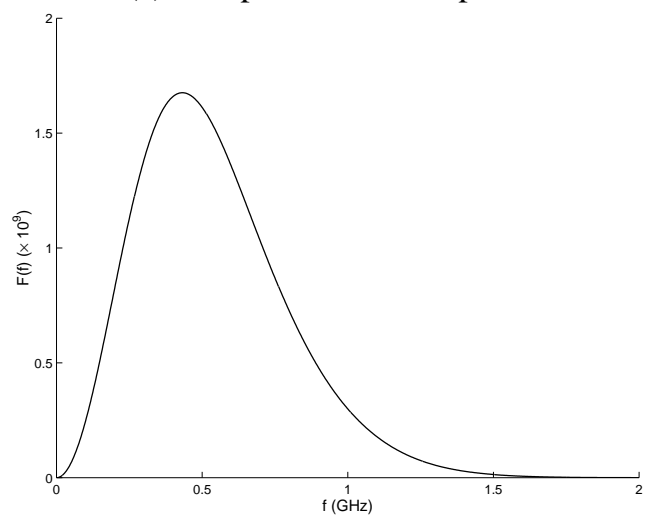

(b) Componentes espectrais

Figura 2. Pulso duplo-gaussiano para $a=0,6$.

\subsubsection{CORRENTES DE FRANJA PARA A COR- REÇÃO DOS CAMPOS DA ÓTICA FÍSICA NO DOMÍNIO DO TEMPO}

As figuras 3-5 mostram o campo espalhado pelo hiperbolóide calculado em pontos de observação localizados a uma distância radial de 100 metros da origem, na região de campo distante dado o espectro predominantemente de alta frequência da excitação, no plano $\mathrm{E}\left(\phi=0^{0}\right)$, e os ângulos de elevação são indicados em cada figura. Para esta análise usouse $n=1$ em (63), de maneira a se obter campos difratados de amplitudes elevadas. As técnicas utilizadas para a determinação do campo espalhado são a TDPO sem correção, determinado a partir de (29), e a TDPO corrigida pelas correntes de franja (TDPO+FWCs), cujo campo irradiado é dado por (38). Para fins de validação da formulação desenvolvida neste trabalho, as respostas da TDPO e da TDPO+FWCs para a componente $\theta$ do campo espalhado pelo refletor são comparadas com a solução de referência (MoM+IFFT) e com a TD-UTD. Nos resultados obtidos é possível distinguir nas parcelas do campo espalhado, a componente do campo refletido, de maior amplitude, e as parcelas devido ao campo difratado, cujo primeiro pulso se superpõe com o pulso refletido à medida em que o ângulo de elevação aumenta e se aproxima da fronteira de reflexão, localizada no ponto $\theta=64^{0}$, como pode ser observado nas Figs. 4 e 5 . Como esperado, observa-se a falta de precisão da TDPO sem correção para as parcelas correspondentes ao campo difratado, o que é 


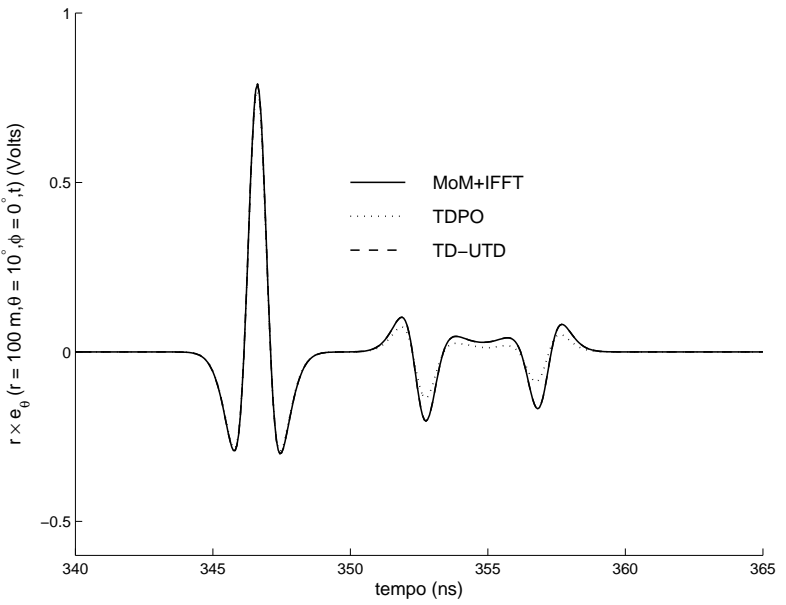

(a) TDPO sem correção

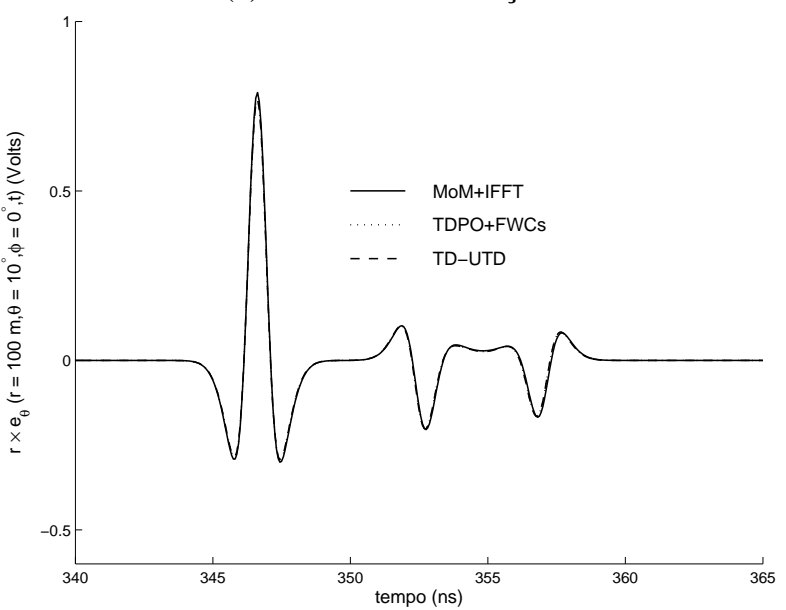

(b) TDPO com correção

Figura 3. Resposta de um hiperbolóide iluminado pelo foco, com lei de iluminação tipo $\cos \theta_{F}$ e comportamento temporal na forma de um pulso duplo-gaussiano, obtida por técnicas de correntes induzidas, para $\theta=10^{0}$.

corrigido quando se utiliza as correntes de franja, obtendo-se uma solução quase indistinguível da solução de referência e da TD-UTD, evidenciando a utilidade das correntes de franja no domínio do tempo.

\subsubsection{CORRENTES EQUIVALENTES DE BORDA PARA A DETERMINAÇÃO DO CAMPO DIFRATADO}

A Fig. 6 mostra a resposta do refletor para um ponto de observação situado sobre seu eixo de simetria, com coordenadas $\left(r=100 \mathrm{~m}, \theta=0^{0}, \phi=0^{0}\right)$. Os campos difratados foram determinados a partir da formulação das TD-EECs apresentada neste trabalho, cujos campos radiados são determinados por (61), e foram somados aos campos da parcela da Ótica Geométrica da TD-UTD [5, 11], compondo a resposta total referenciada como TD-GO+EECs. O resultado assim obtido é comparado com a solução de referência (MoM+IFFT) e também com uma versão da TD-GO+EECs cuja formulação de correntes equivalentes emprega coeficientes angulares sem

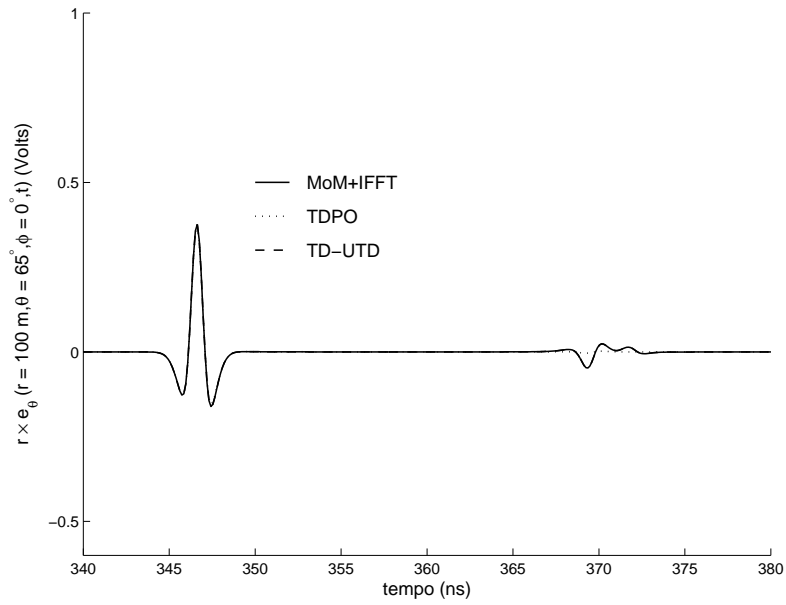

(a) TDPO sem correção

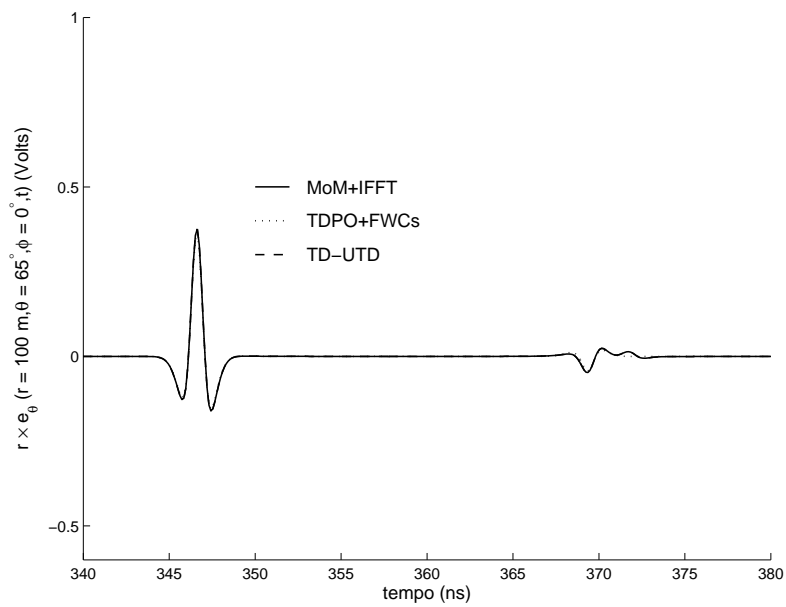

(b) TDPO com correção

Figura 4. Resposta de um hiperbolóide iluminado pelo foco, com lei de iluminação tipo $\cos \theta_{F}$ e comportamento temporal na forma de um pulso duplo-gaussiano, obtida por técnicas de correntes induzidas, para $\theta=65^{\circ}$.

extração de singularidades [1]. É possível perceber no resultado apresentado que o primeiro pulso do campo espalhado corresponde à parcela refletida, enquanto que o segundo está associado ao campo difratado pelas bordas do refletor. No caso dos métodos de análise no domínio temporal, pode-se afirmar, para o resultado sob análise, que o primeiro pulso é determinado pela TD-GO, enquanto que o segundo pulso é obtido pela formulação das correntes equivalentes de borda. Tal fato é prontamente verificado ao se observar os retardos de tempo associados a cada pulso mostrado na figura. $\mathrm{O}$ primeiro pulso tem o menor retardo uma vez que percorre o menor caminho entre a fonte e o ponto de observação, enquanto que o segundo pulso consiste da soma dos campos difratados, que percorrem um caminho maior e chegam ao mesmo tempo no observador. Observa-se a concordância entre os resultados das três técnicas, o que é esperado uma vez que o ponto de observação está suficientemente afastado das fronteiras de sombra do refletor, de modo que não há singularides nas parcelas das correntes equivalentes.

A importância da extração das singularidades das correntes equivalentes se torna evidente ao se analisar o campo espal- 
Correntes equivalentes de borda para a análise da resposta de antenas refletoras excitadas por pulsos, parte II: formulação no domínio do tempo e aplicações

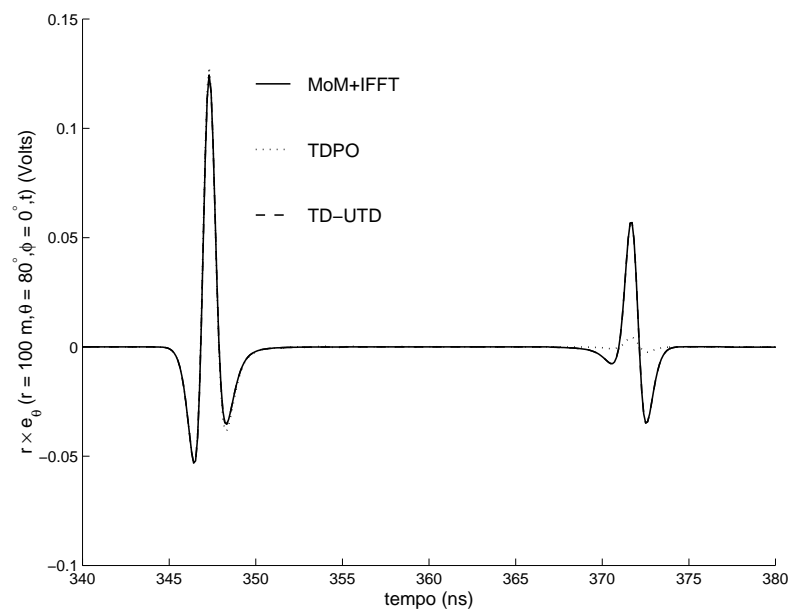

(a) TDPO sem correção

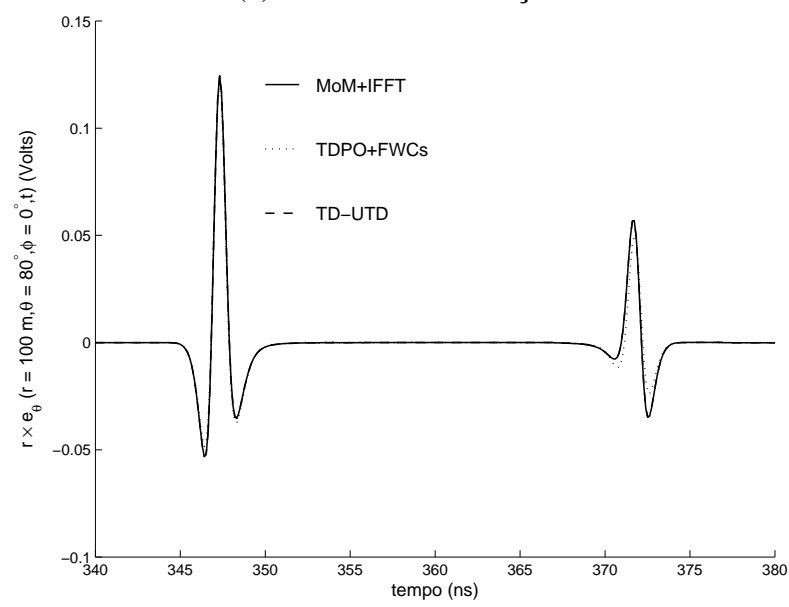

(b) TDPO com correção

Figura 5. Resposta de um hiperbolóide iluminado pelo foco, com lei de iluminação tipo $\cos \theta_{F}$ e comportamento temporal na forma de um pulso duplo-gaussiano, obtida por técnicas de correntes induzidas, para $\theta=80^{\circ}$.

hado para um ponto de observação localizado nas proximidades de uma das fronteiras de sombra do refletor, onde é conhecido que há uma singularidade nas correntes equivalentes de borda, a qual pode ser extraída. A Fig. 7 mostra os campos espalhados da solução de referência e das duas versões para a TD-GO+EECs no ponto $\left(r=100 \mathrm{~m}, \theta=65^{0}, \phi=0^{0}\right)$, o qual está muito próximo da fronteira de sombra, localizada em $\theta=64^{0}$. Neste caso, observa-se que a versão das correntes equivalentes com singularidades extraídas mantém a regularidade, enquanto que a versão que contém as singularidades diverge.

Como foi comentado anteriormente, é sabido que a simetria circular do refletor analisado aqui leva à existência de uma cáustica para o campo difratado, localizada sobre seu o eixo de simetria, o que inviabiliza a aplicação dos métodos de rastreamento de raios no cálculo do campo espalhado para pontos de observação próximos a ele. As Figs. 8 e 9 mostram a utilidade das correntes equivalentes de borda para a solução desta dificuldade. Neste caso, são apresentadas comparações entre as respostas dos campos espalhados da TDPO+FWCs, TD-GO+EECs e da TD-UTD, determinados

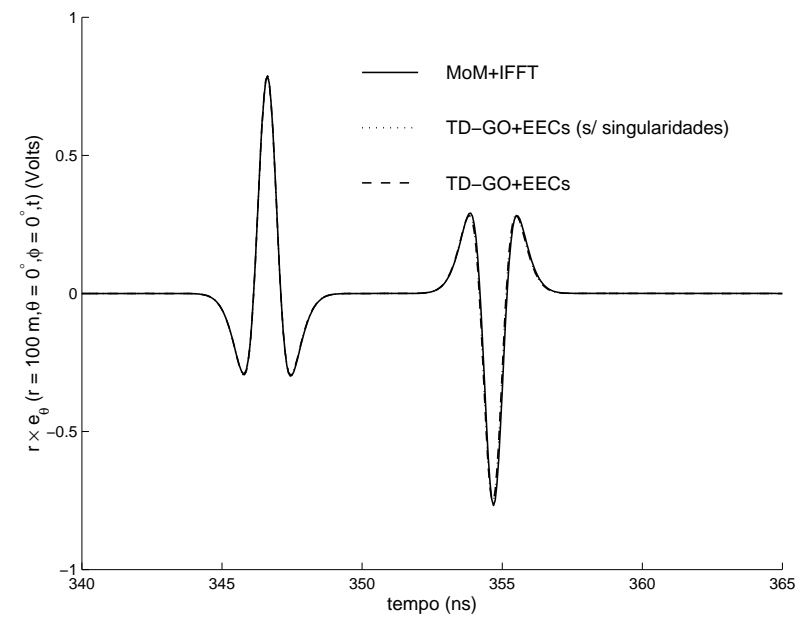

Figura 6. Resposta de um hiperbolóide iluminado pelo foco por um pulso duplo-gaussiano, para $\theta=0^{0}$.

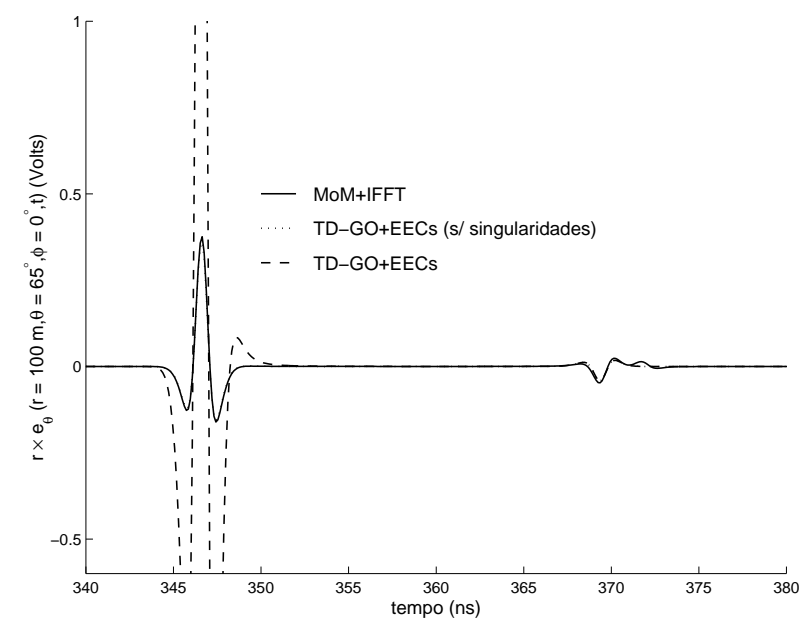

Figura 7. Resposta de um hiperbolóide iluminado pelo foco por um pulso duplo-gaussiano, para $\theta=65^{\circ}$.

em pontos de observação com coordenada radial $r=100$ metros, ângulos de elevação $\theta=0^{0}$ e $\theta=2^{0}$, e ângulo de azimute $\phi=0^{0}$, situados bem próximo ao eixo de simetria do hiperbolóide em $\theta=0^{0}$. É possível verificar a regularidade das respostas das técnicas de correntes equivalentes, TDPO+FWCs e TD-GO+EECs, enquanto o método da TD-UTD apresenta resultados errôneos, esperados para esta região de observação. Para este estudo de caso em particular, a aplicação da TD-GO+EECs é mais vantajosa em comparação à Ótica Física corrigida por correntes de franja, uma vez que a combinação da Ótica Geométrica com correntes de borda permite uma computação mais rápida.

\subsection{PARABOLÓIDE ILUMINADO PELO FOCO}

Neste estudo de caso, analisa-se a resposta de um parabolóide de revolução com diâmetro de 7,5 m e razão distância focal/diâmetro $F / D=0,4$, também excitado por um pulso duplo gaussiano, cujo comportamento temporal é expresso em (62), de maneira que o campo incidente tem 


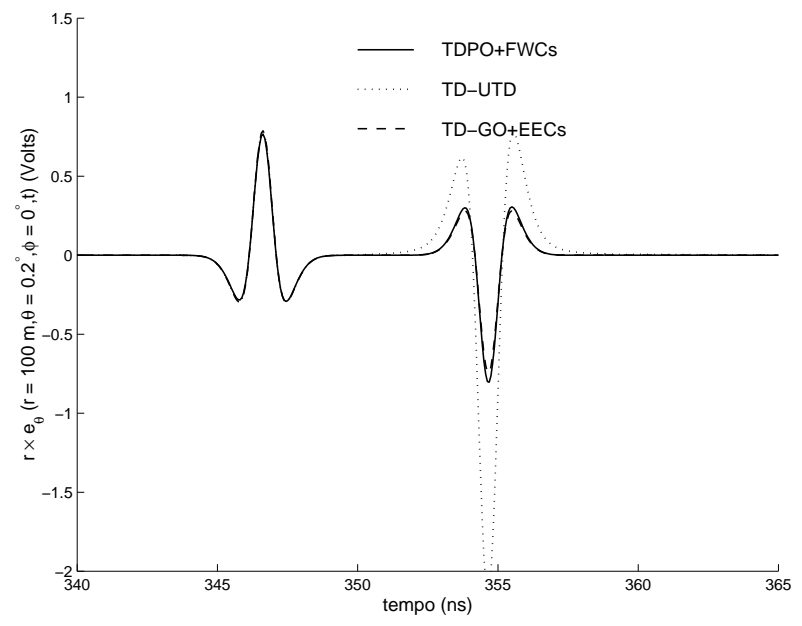

Figura 8. Resposta de um hiperbolóide iluminado pelo foco, com lei de iluminação tipo $\cos \theta_{F}$ e comportamento temporal na forma de um pulso duplo-gaussiano para $\theta=0,2^{0}$ : comparação entre técnicas óticas e de correntes equivalentes.

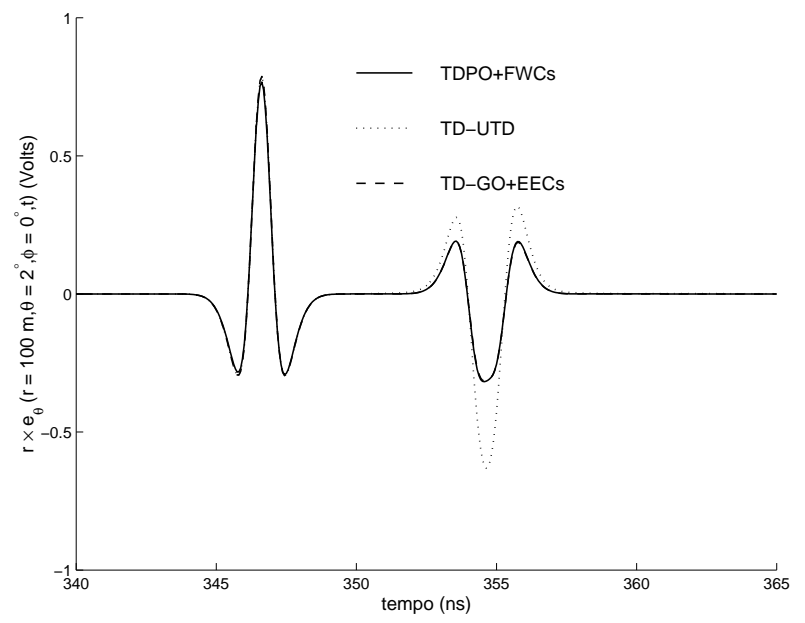

Figura 9. Resposta de um hiperbolóide iluminado pelo foco, com lei de iluminação tipo $\cos \theta_{F}$ e comportamento temporal na forma de um pulso duplo-gaussiano para $\theta=2^{0}$ : comparação entre técnicas óticas e de correntes equivalentes.

a forma indicada em (63). Aqui foi utilizado um valor de $a=0,2 \mathrm{~m}$, de maneira que o pulso tem suas componentes espectrais distribuídas em torno de $1,3 \mathrm{GHz}$, como pode ser observado na Fig. 10.

\subsubsection{CORRENTES DE FRANJA PARA A COR- REÇÃO DE CAMPOS DA TDPO}

As Figs. 11 e 12 retratam o campo espalhado pelo refletor parabolólico em pontos de observação de coordenada radial $r=100 \mathrm{~m}$, ângulos de elevação $\theta=0^{0}$ e $\theta=40^{\circ}$, respectivamente, e ângulo de azimute $\phi=0^{0}$. A exemplo do que foi feito anteriormente para o hiperbolóide, comparou-se as respostas obtidas pela aplicação da TDPO sem correção, TDPO+FWCs, TD-UTD (apenas para $\theta=40^{\circ}$ ) e a solução de referência, MoM+IFFT. Observa-se que na resposta para $\theta=0^{0}$ tem-se um único pulso, uma vez que sobre o eixo

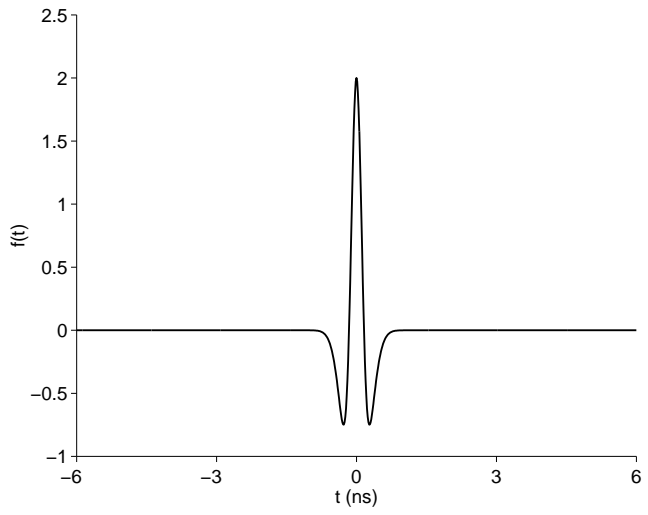

(a) Comportamento temporal

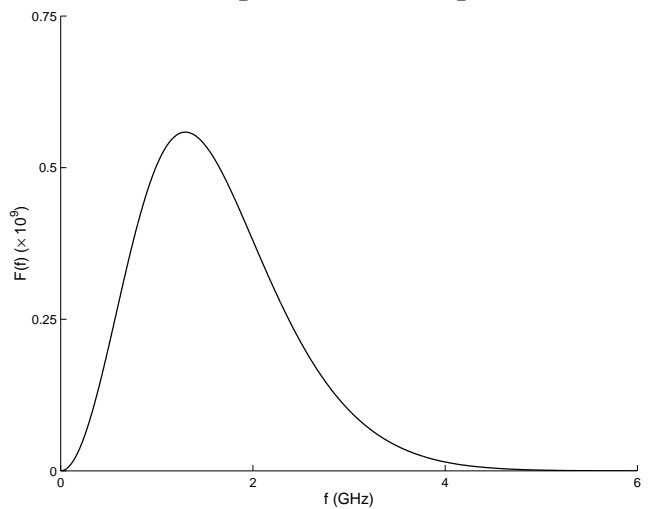

(b) Componentes espectrais

Figura 10. Pulso duplo-gaussiano para $a=0,2$.

de simetria do refletor os campos difratados percorrem a mesma distância até o observador. Para $\theta=40^{\circ}$ têm-se dois pulsos correspondendo às parcelas de energia difratada, a qual percorre caminhos diferentes até o ponto de observação. Mais uma vez, é possível evidenciar a aplicabilidade dos métodos de análise no domínio do tempo, observando-se a concordância das respostas obtidas através de sua aplicação com o que advém da solução de referência. Verifica-se que, no caso do parabolóide, a exemplo do que acontece na análise no domínio da frequência, a correção das correntes da Ótica Física só apresenta resultados significativos para pontos de observação bastante afastados do eixo de simetria do refletor (Fig. 12).

\subsubsection{CORRENTES EQUIVALENTES DE BORDA PARA O CÁLCULO DO CAMPO DIFRATADO}

O potencial das correntes equivalentes de borda para o cálculo do campo difratado por antenas refletoras é demonstrado para este caso em estudo analisando-se a resposta do parabolóide para pontos de observação com coordenadas radial e azimutal iguais a $r=100 \mathrm{~m}$ e $\phi=0^{0}$, respectivamente, com ângulos de elevação pertencentes ao intervalo $\left[20^{0}, 180^{\circ}\right]$. As figuras 13-16 mostram o campo espalhado pelo parabolóide, determinado pelas correntes equivalentes de borda, cujos campos radiados são expressos por (61), comparando-se os resultados obtidos com a solução de referência. Nos pontos de observação escolhidos para esta análise 
Correntes equivalentes de borda para a análise da resposta de antenas refletoras excitadas por pulsos, parte II: formulação no domínio do tempo e aplicações

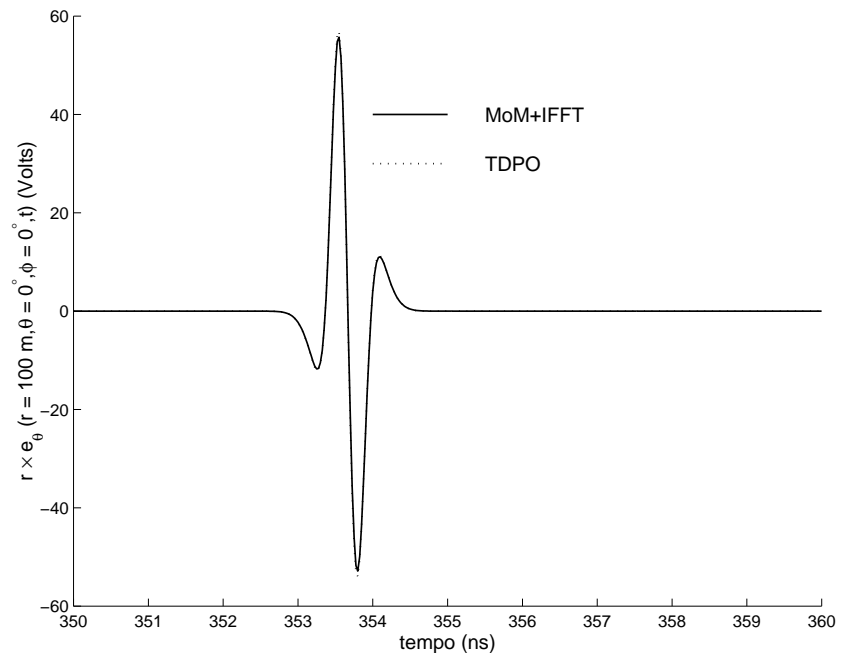

(a) TDPO sem correção

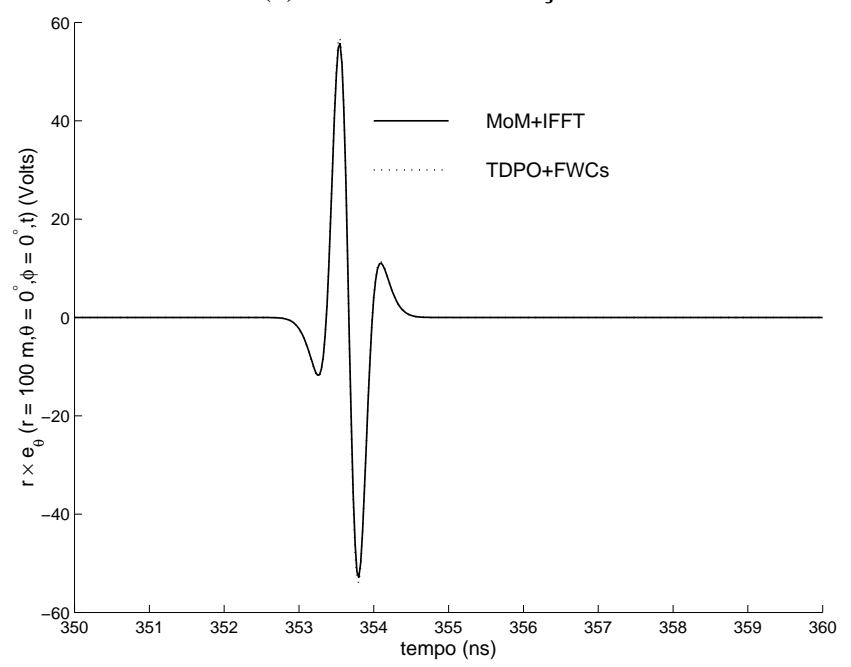

(b) TDPO com correção

Figura 11. Resposta de um parabolóide iluminado pelo foco, com lei de iluminação tipo $\cos \theta_{F}$ e comportamento temporal na forma de um pulso duplo-gaussiano, obtida por técnicas de correntes induzidas, para $\theta=0^{0}$.

obtém-se um campo espalhado no forma de dois pulsos separados por um intervalo de tempo que aumenta até atingir seu valor máximo em $\theta=90^{\circ}$, onde a diferença de caminho percorrido pelos pulsos difratados é igual ao diâmetro do refletor. Para ângulos de elevação no intervalo $\left[90^{\circ}, 180^{\circ}\right]$ a diferença de tempo entre os pulsos difratados diminui até a sua superposição nas costas do refletor (Fig. 16).

Os resultados apresentados nas Figs. 13-16 mostram mais uma vez a precisão das correntes equivalentes de borda, que se constituem em uma técnica robusta para a determinação do campo difratado para qualquer ponto de observação, uma vez que a aplicação da formulação da equação (61) não envolve uma integração sobre toda a superfície do refletor como acontece com a Ótica Física, o que acarreta em um menor tempo de computação neste caso. Além disso, não se faz necessário analisar as posições relativas entre pontos de difração e de observação para verificar se há bloqueio de raios difratados pelo refletor, como aconteceria na aplicação de métodos de rastreamento de raios para o cálculo dos
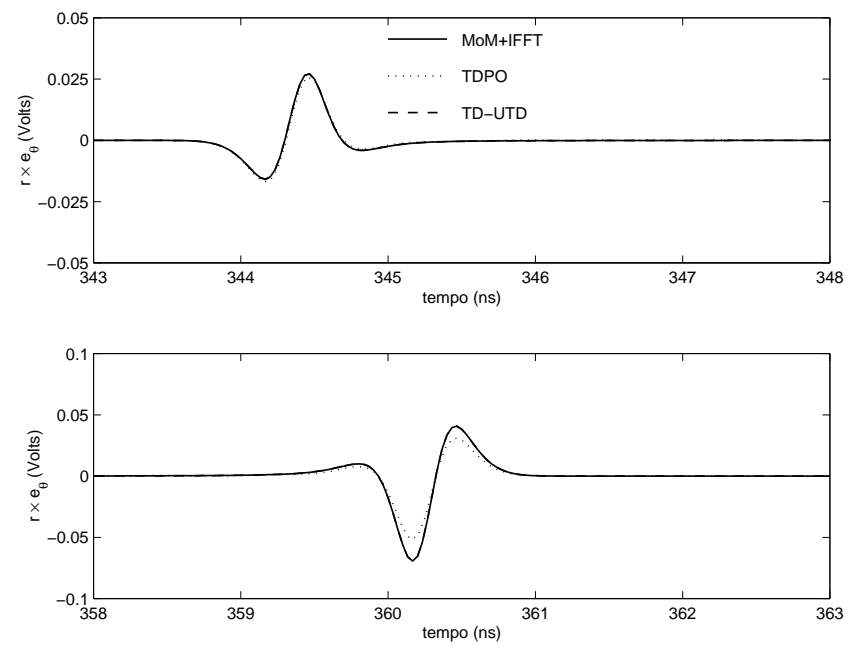

(a) TDPO sem correção
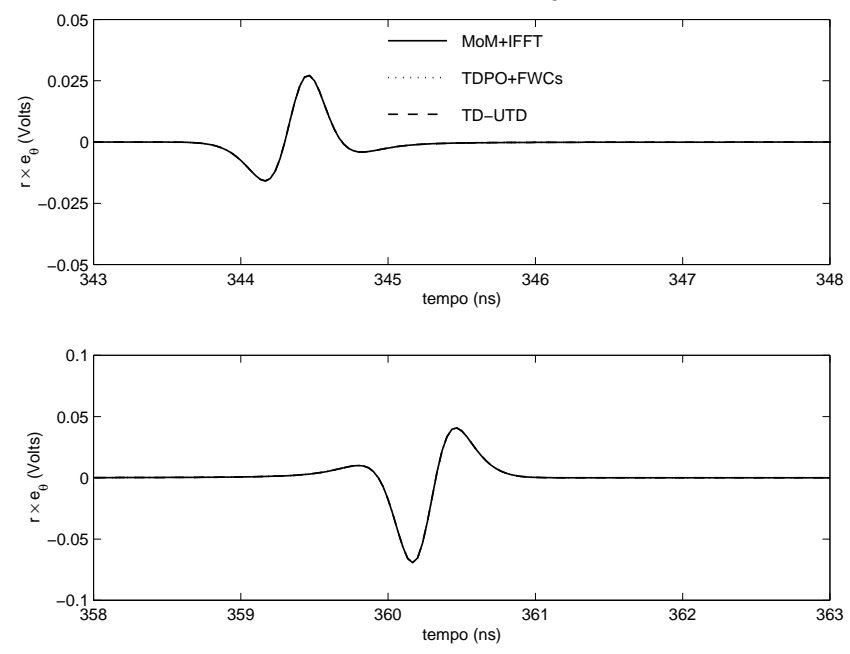

(b) TDPO com correção

Figura 12. Resposta de um parabolóide iluminado pelo foco, com lei de iluminação tipo $\cos \theta_{F}$ e comportamento temporal na forma de um pulso duplo-gaussiano, obtida por técnicas de correntes induzidas, para $\theta=40^{\circ}$.

campos para ângulos de elevação pertencentes ao intervalo $\left[90^{\circ}, 180^{\circ}\right]$.

\section{CONCLUSÕES}

O presente artigo introduziu formulações temporais de correntes equivalentes de borda em superfícies condutoras, endereçadas à análise da resposta transiente de antenas refletoras excitadas por fontes pulsadas. As equações aqui deduzidas permitem a determinação de campos eletromagnéticos irradiados, regulares em todo o espaço de observação, os quais podem ser utilizados tanto para a correção da resposta obtida pela aplicação da Ótica Física (TDPO), quanto para o cálculo de campos difratados em pontos de observação localizados em regiões de focalização (cáusticas), típicas de antenas colimadoras de energia. A metodologia empregada para o estabelecimento da formulação envolve a inversão de Fourier e o uso de representações analíticas de sinais temporais aplicadas 

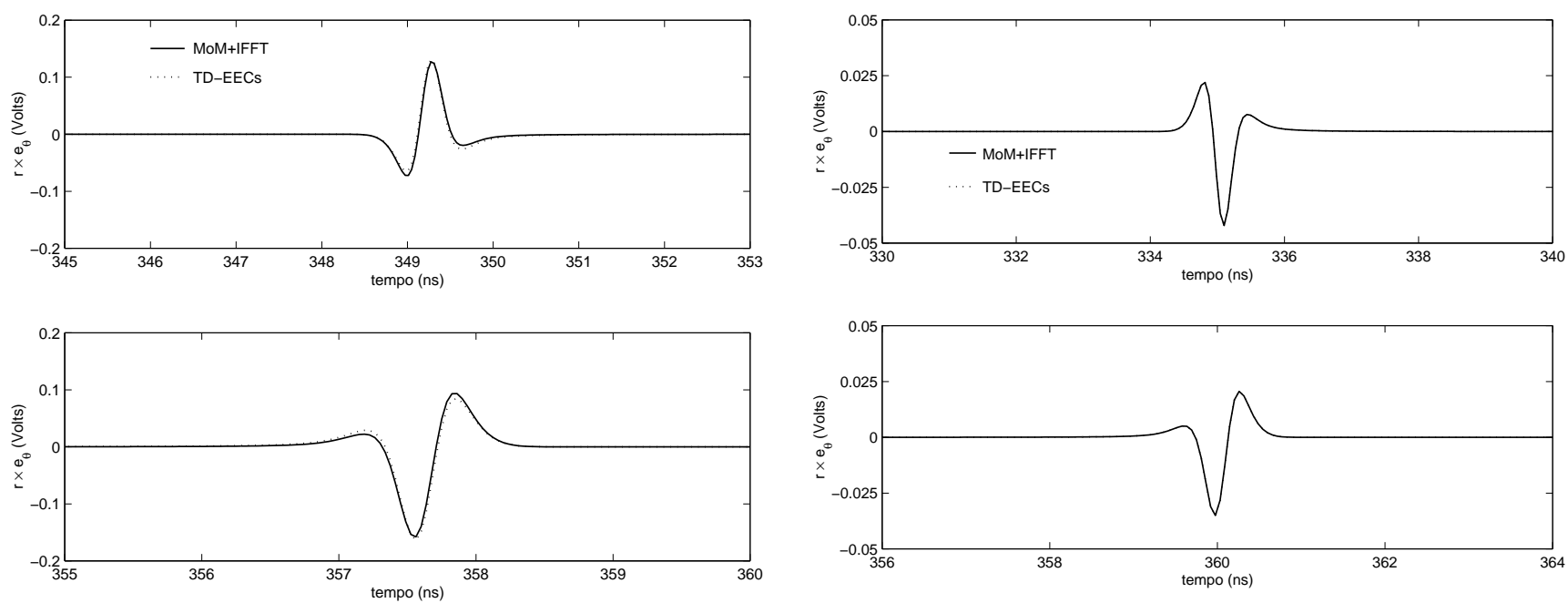

Figura 13. Resposta de um parabolóide iluminado pelo foco por um pulso duplo-gaussiano, para $\theta=20^{\circ}$.
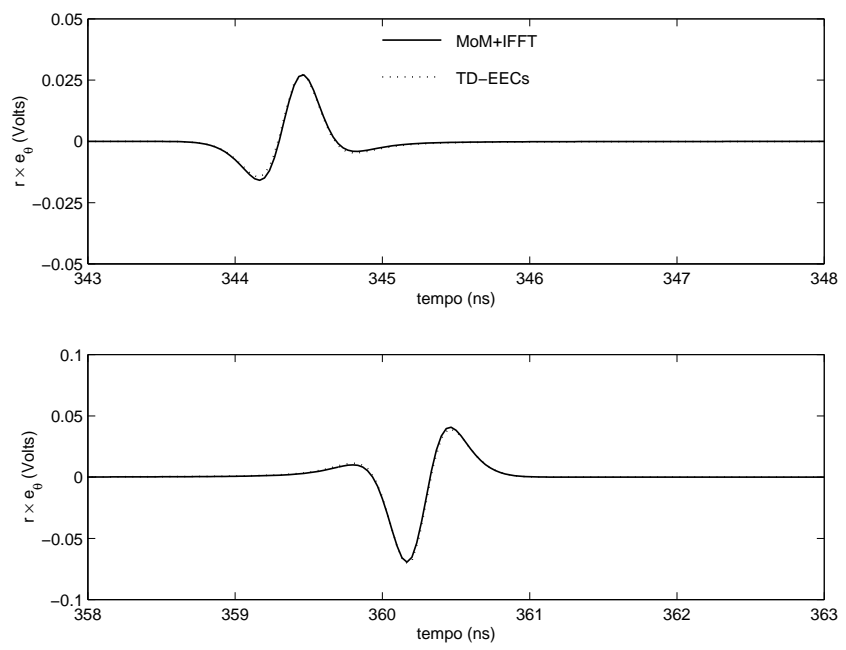

Figura 14. Resposta de um parabolóide iluminado pelo foco por um pulso duplo-gaussiano, para $\theta=40^{\circ}$.

a equações de correntes equivalentes de borda do domínio da frequência, as quais foram revisadas, incluindo-se aí a extração de suas singularidades [5]. Esta metodologia adotada garantiu a robustez das correntes equivalentes aqui deduzidas, conforme evidenciado pelos resultados apresentados para diversas aplicações.

No que concerne a aplicação das formulações desenvolvidas neste artigo à solução de problemas de antenas refletoras, uma extensão natural deste trabalho é o estudo da resposta transiente das mesmas operando com excitações de comportamento temporal governado por sinais de modulação digital. Uma primeira abordagem neste sentido foi a análise de refletores parabólicos iluminados por sinais com modulação BPSK utilizando-se bits extremamente curtos feita por Sun $[14,15]$, onde se usou a TDPO para a determinação da resposta transiente, a partir da qual se extraiu informações importantes para o projeto de sistemas de radar, tais como a estimação da relação entre a largura de bits e o comprimento de código utilizado. A análise feita naquele trabalho investigou aspectos importantes da resposta transiente, associados à iluminação dos refletores e desfocalização de fontes, pois a

Figura 15. Resposta de um parabolóide iluminado pelo foco por um pulso duplo-gaussiano, para $\theta=90^{\circ}$.

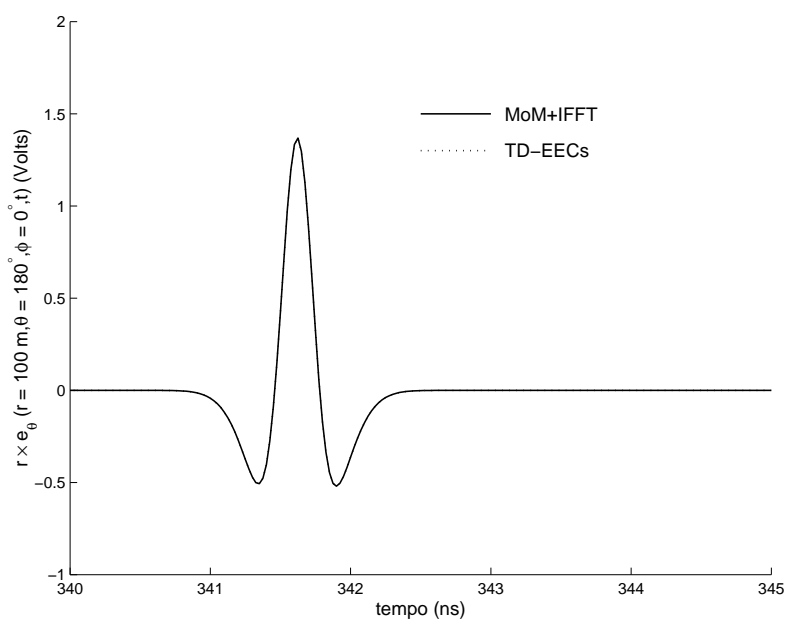

Figura 16. Resposta de um parabolóide iluminado pelo foco por um pulso duplo-gaussiano, para $\theta=180^{\circ}$.

excitação de uma antena refletora por pulsos periódicos deve ser feita de forma que o estado estacionário ocorra, o que possibilita manter-se uma baixa dispersão da forma de onda a ser transmitida. As técnicas assintóticas desenvolvidas neste trabalho permitem a determinação da resposta transiente de antenas refletoras excitadas pelo mesmo tipo de fonte utilizado na referência mencionada acima, tendo como vantagens a inclusão de efeitos de difração em bordas e sendo flexíveis para a aplicação em configurações mais complexas, tais como sistemas duplo refletor e refletores modelados. A aplicação mencionada acima vem sendo implementada e alguns resultados preliminares já foram obtidos [28]-[30], de modo que esta será tema de publicações futuras.

\section{REFERÊNCIAS}

[1] A. Michaeli, "Equivalent edge currents for arbitrary aspects of observation", IEEE Transactions on Antennas and Propagation, vol. 32, no. 3, pp. 252-258, March 1984.

[2] A. Michaeli, "Correction to "Equivalent edge currents for arbitrary aspects of observation", IEEE Transactions on Antennas and Propagation, vol. 33, no. 2, pp. 227, February 1985. 
[3] A. Michaeli, "Elimination of infinities in equivalent edge currents, part I: fringe current components", IEEE Transactions on Antennas and Propagation, vol. 34, no. 7, pp. 912-918, July 1986.

[4] A. Michaeli, "Elimination of infinities in equivalent edge currents, part II: physical optics components", IEEE Transactions on Antennas and Propagation, vol. 34, no. 8, pp. 1034-1037, August 1986.

[5] C. G. Rego, Formulações assintóticas para o espalhamento por superficies condutoras no domínio do tempo e aplicações à análise de transientes em antenas refletoras, Tese de Doutorado, Departamento de Engenharia Elétrica, Centro de Estudos em Telecomunicações, Pontifícia Universidade Católica do Rio de Janeiro, Agosto de 2001.

[6] M. Z. Win and R. A. Scholtz, "Ultra-wide bandwidth timehopping spread-spectrum impulse radio for wireless multipleaccess communications", IEEE Transactions on Communications, vol. 48, no. 4, pp. 679-691, April 2000.

[7] C. G. Rego, F. J. V. Hasselmann and F. J. S. Moreira, "Time-domain analysis of a reflector antenna illuminated by a Gaussian pulse", in Proceedings of SBMO/IEEE International Microwave an Optoelectronics Conference, Rio de Janeiro, RJ, Brasil, Agosto de 1999, pp. 534-537.

[8] C. G. Rego, F. J. V. Hasselmann and F. J. S. Moreira, "Timedomain analysis of of a reflector antenna illuminated by a Gaussian pulse", Journal of Microwaves and Optoelectronics, vol. 1, no. 1, pp. 20-28, September 1999.

[9] C. G. Rego, F. J. V. Hasselmann and F. J. S. Moreira, "A comparison between asymptotic methods for time-domain analysis of reflector antennas", in Proceedings of COMPUMAG - 12th Conference on the computation of electromagnetic fields, Sapporo, Japan, October 1999, pp. 152-153.

[10] C. G. Rego and F. J. V. Hasselmann, "Time-domain analysis of pulse-excited reflector antennas", in Proceedings of IEEE Antennas and Propagation Society International Symposium, Salt Lake City, Utah, USA, July 2000, pp. 2046-2049.

[11] C. G. Rego, F. J. V. Hasselmann e F. J. S. Moreira, "Análise no domínio do tempo de antenas refletoras excitadas por pulsos", in Anais do XI Simpósio Brasileiro de Microondas e Optoeletrônica, João Pessoa, PB, Agosto de 2000, pp.329-333.

[12] C. G. Rego and F. J. V. Hasselmann, "Time-domain analysis of pulse-excited reflector antennas - UAT approach", in Proceedings of IEEE Antennas and Propagation Society International Symposium, Boston, Massachussets, USA, July 2001, pp. 376379.

[13] C. G. Rego and F. J. V. Hasselmann, "Time-domain 3-D vector analysis of pulse-excited perfectly conducting wedges: the UAT approach", in Proceedings of SBMO/IEEE International Microwave an Optoelectronics Conference, Belém, PA, Brasil, August 2001, pp. 417-420.

[14] E.-Y. Sun, Time-domain analysis of reflector antennas and its applications, $\mathrm{PhD}$ thesis, Department of Electrical EngineeringElectrophysics, University of Southern California, May 1991.

[15] E.-Y. Sun and W. V. T. Rusch, "Time-domain physical-optics", IEEE Transactions on Antennas and Propagation, vol. 42, no. 1, pp. 9-15, January 1994.

[16] E. Heyman and L. B. Felsen, "Weakly dispersive spectral theory of transients, part I: formulation and interpretation", IEEE Transactions on Antennas and Propagation, vol. 35, no. 1, pp. 80-86, January 1987.

[17] E. Heyman and L. B. Felsen, "Weakly dispersive spectral theory of transients, part II: evaluation of the spectral integral", IEEE Transactions on Antennas and Propagation, vol. 35, no. 5, pp. 574-580, May 1987.

[18] E. Heyman and L. B. Felsen, "Weakly dispersive spectral the- ory of transients, part III: applications", IEEE Transactions on Antennas and Propagation, vol. 35, no. 11, pp. 1258-1266, November 1987.

[19] R. Ianconescu and E. Heyman, "Pulse field diffraction by a perfectly conducting wedge: a spectral theory of transients analysis", IEEE Transactions on Antennas and Propagation, vol. 42, no. 6, pp. 781-789, June 1994.

[20] L. B. Felsen, "Diffraction of a pulse field from an arbitrarily oriented electric or magnetic dipole by a perfectly conducting wedge", SIAM J. Appl. Math., vol. 26, no. 2, pp. 306-312, March 1974.

[21] P. M. Johansen, "Time-domain version of the physical theory of diffraction," IEEE Transactions on Antennas and Propagation, vol. 47, no. 2, pp. 261-270, February 1999.

[22] S. Altintas and P. Russer, "Time-domain equivalent edge currents for transient scattering," IEEE Transactions on Antennas and Propagation, vol. 49, no. 4, pp. 602-606, April 2001.

[23] P. R. Rosseau and P. H. Pathak, Time-domain version of the uniform geometrical theory of diffraction, Technical Report 721564-3, The Ohio State University ElectroScience Laboratory, 1996.

[24] R. N. Bracewell, The Fourier transform and its applications, McGraw-Hill International Editions, Singapore, 1986.

[25] L. B. Felsen and N. Marcuvitz, Radiation and Scattering of Waves, Englewood Cliffs, NJ: Prentice, 1973.

[26] L. B. Felsen, "Propagation and diffraction of transient fields in non-dispersive and dispersive media", in Transient Electromagnetic Fields, L. B. Felsen, Editor, chapter 1, pp. 1-72, Springer Verlag, Berlin, 1976.

[27] P. Y. Ufimtsev, "Method of edge waves in the physical theory of diffraction", translation from Russian prepared by the U.S. Air Force Foreign Technology Division, Wright-Patterson AFB, Ohio, released for public distribuition, September 1971.

[28] C. G. Rego, S. T. M. Gonçalves, W. C. P. dos Anjos e F. J. V. Hasselmann, "Resposta transiente de antenas refletoras aplicadas em sistemas de comunicação digital", in Anais do XI Simpósio Brasileiro de Microondas, São Paulo, SP, Brasil, Agosto de 2004, pp. 417-422.

[29] C. G. Rego, F. J. V. Hasselmann, S. T. M. Gonçalves, W. C. P. dos Anjos e E. L. Marques, "Elementos para a caracterização temporal de antenas refletoras", in Anais do XI Simpósio Brasileiro de Microondas, São Paulo, SP, Brasil, Agosto de 2004, pp. 525-530.

[30] C. G. Rego, F. J. V. Hasselmann, S. T. M. Gonçalves, and E. L. Marques, "A high-frequency formulation for temporal characterization of reflector antennas", accepted for presentation on 2005 IEEE/ACES International Conference on Wireless Communications and Applied Computational Electromagnetics, Honolulu, Hawaii, USA, April 2005.

Cássio Gonçalves do Rego recebeu o diploma de Engenheiro Eletricista pela Universidade de Brasília em 1988, Mestre em Ciências em Engenharia Elétrica e Doutor em Ciências em Engenharia Elétrica pela Pontifícia Universidade Católica do Rio de Janeiro em 1991 e 2001, respectivamente. É coordenador do Grupo de Antenas, Propagação e Teoria Eletromagnética (GAPTEM) da Universidade Federal de Minas Gerais. Seus principais interesses em pesquisa são em métodos assintóticos em eletromagnetismo (domínios do tempo e da frequência), antenas e propagação de ondas de rádio.

Flávio José Vieira Hasselmann obteve os diplomas de Engenheiro Eletricista (Telecomunicações) e Mestre em Ciências em Engenharia Elétrica pela Pontifícia Universidade Católica do Rio de Janeiro em 1974 e 1976, respectivamente, e de Doctor of Phylosophy (Electrophysics) pelo Polytechnic Institute of New York (hoje, 
Polytechnic University) em 1980. É diretor do CETUC (Centro de Estudos em Telecomunicações da PUC/Rio) e chairman do Capítulo Conjunto Rio de Janeiro das Sociedades AP/MTT/ED do IEEE. Na SBrT, foi membro do Conselho Deliberativo no período 19831985, coordenador técnico do IV Simpósio Brasileiro de Telecomunicações e editor da área de antenas e propagação da revista da SBrT no período 1987-1991. Seus principais interesses em pesquisa são em métodos assintóticos em eletromagnetismo, antenas refletoras e espalhamento em cobertura de sistemas celulares. 